\title{
Channelling inflammation: gasdermins in physiology and disease
}

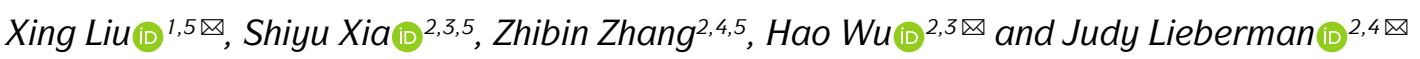

Abstract | Gasdermins were recently identified as the mediators of pyroptosis - inflammatory cell death triggered by cytosolic sensing of invasive infection and danger signals. Upon activation, gasdermins form cell membrane pores, which release pro-inflammatory cytokines and alarmins and damage the integrity of the cell membrane. Roles for gasdermins in autoimmune and inflammatory diseases, infectious diseases, deafness and cancer are emerging, revealing potential novel therapeutic avenues. Here, we review current knowledge of the family of gasdermins, focusing on their mechanisms of action and roles in normal physiology and disease. Efforts to develop drugs to modulate gasdermin activity to reduce inflammation or activate more potent immune responses are highlighted.

\section{Gasdermins \\ A family of homologous pore-forming proteins whose N-terminal fragments permeabilize cell membranes when the protein is cleaved in the linker region connecting the active $\mathrm{N}$-terminal and inhibitory C-terminal domains.}

'The Center for Microbes, Development and Health, Key Laboratory of Molecular Virology and Immunology, Institut Pasteur of Shanghai, Chinese Academy of Sciences, Shanghai, China.

${ }^{2}$ Program in Cellular and Molecular Medicine, Boston Children's Hospital, Boston, MA, USA.

${ }^{3}$ Department of Biological Chemistry and Molecular Pharmacology, Harvard Medical School, Boston, MA, USA.

${ }^{4}$ Department of Pediatrics, Harvard Medical School, Boston, MA, USA.

${ }^{5}$ These authors contributed equally: Xing Liu, Shiyu Xia, Zhibin Zhang

凶e-mail: xingliu@ips.ac.cn; wu@crystal.harvard.edu; judy.lieberman@ childrens.harvard.edu https://doi.org/10.1038/ s41573-021-00154-z
Gasdermins (GSDMs) are a recently characterized protein family encoded by six paralogous genes: GSDMA, GSDMB, GSDMC, GSDMD, GSDME (also known as DFNA5) and DFNB59 (also known as pejvakin) ${ }^{1}$. Proteolytic cleavage of the GSDMs liberates an N-terminal (NT) fragment, which can assemble in membranes to form pores (verified for most, but not all, of the family). GSDM pores can disrupt cell membrane integrity to trigger an inflammatory cell death in which cellular contents, including inflammatory cytokines, are released into the extracellular space ${ }^{2-4}$ (BOX 1; FICS 1,2). GSDM-mediated cell death is termed pyroptosis (fiery death).

The name 'gasdermin' comes from GSDM expression in the gastroinstestinal tract and skin. Individual GSDMs are selectively expressed with varying abundance wherever the body encounters, detects and responds to infection ${ }^{3,5}$, being predominantly expressed in specific mucosal sites (TABLE 1). GSDMA is found in skin and the gastrointestinal tract, GSDMB is located in the lung, oesophagus, gastrointestinal tract and immune cells, GSDMC is found in keratinocytes and the gastrointestinal tract, and GSDMD is found in the gastrointestinal epithelia and in the sentinel cells of the immune system, macrophages and dendritic cells $s^{3}$. GSDME has a different pattern of expression in mesenchymal cells muscle (both skeletal and cardiac), central nervous system (CNS) and placenta ${ }^{6}$. The physiological role of GSDME in these tissues, where inflammation might be harmful, is not entirely clear, although it may be involved in development ${ }^{\text {? }}$.

GSDMs A-E share highly conserved NT and C-terminal (CT) domains separated by a variable linker (FIG. 3a). The NT domain of GSDMs binds to acidic phospholipids in the inner leaflet of cell membranes and forms pores $^{8-11}$. In the full-length protein, the CT domain folds back on the NT domain to auto-inhibit pore formation ${ }^{9,12}$. GSDM cleavage in the linker region liberates the active, pore-forming NT domain. GSDMD is the most studied GSDM as it is the key executioner of inflammasome-induced pyroptosis and is probably the most important GSDM mediating disease-associated inflammation. GSDMD cleavage and pyroptosis are activated when cytosolic danger and infection sensors in macrophages and dendritic cells assemble into large supramolecular complexes called inflammasomes that recruit and activate inflammatory caspases ${ }^{13-15}$. The inflammatory caspases activate GSDMD by cleaving after Asp275 (mouse Asp276) in the linker ${ }^{13,14}$. By contrast, GSDME is activated during classical apoptosis (BOX 2), by caspase 3 cleavage after Asp270, which converts noninflammatory apoptotic death into inflammatory pyroptotic death in cells that express GSDME ${ }^{16,17}$. Granzymes (Gzms) $\mathrm{A}$ and $\mathrm{B}$, death-inducing proteases in natural killer $(\mathrm{NK})$ cells and cytotoxic T lymphocytes (CTLs), also cleave GSDMB and GSDME, respectively, to activate pyroptosis during killer cell attack, which was previously thought of as noninflammatory ${ }^{18,19}$. A recent study showed that GSDMC can be cleaved by caspase 8 after TNF-mediated death receptor signalling to trigger pyroptosis ${ }^{20}$. How GSDMA is activated is currently unknown.

GSDM pores in the plasma membrane act as channels through which low-molecular-weight cellular contents are released into the extracellular space to initiate inflammation (FIC. 2). Importantly, the pores mediate the 'unconventional protein secretion' of the pro-inflammatory cytokines (IL-1 $\beta$ and IL-18) that lack a signal peptide for secretion via the endoplasmic reticulum to Golgi secretory pathway ${ }^{21}$. Cellular alarmins, including ATP 
Pyroptosis

A programmed inflammatory the cleavage and activation of a gasdermin, leading to loss of cell membrane integrity and release of pro-inflammatory cytokines and cellular proteins that can act as danger signals.

Inflammasome Intracellular complex of infection that recognize pathogen-associated molecular patterns (PAMPs) or of danger that recognize damageassociated molecular patterns (DAMPs) and then assemble into supramolecular complexes that activate inflammatory caspases; the canonical inflammasome activates caspase 1 and the noncanonical inflammasome activates caspase 4 or caspase 5 in humans or caspase 11 in mice.

Inflammatory caspases Cysteine proteases that are activated by proximity-induced autocatalytic cleavage when they dimerize after recruitment to canonical (caspase 1) or noncanonical (caspase 4, 5 or 11) inflammasomes and are responsible for processing inflammatory cytokines and gasdermin D to induce pyroptosis or hyperactivation.

Apoptosis

A noninflammatory pro-

grammed cell death mediated by mitochondrial outer membrane permeabilization and activation of the effector caspases (3, 6 and 7). cell death, which occurs after cytosolic sensors of invasive

and HMGB1, cleaved GSDMs and other cytokines and chemokines, are released at the same time to amplify inflammation in the tissue and recruit immune cells to respond to the infection or damage. Many small molecules and other proteins, which may differ between cell types, are probably released from pyroptotic cells. Pore formation triggers rapid pyroptotic cell death in which the damaged membrane forms large ballooning bubbles and dying cells appear to flatten as their cytoplasmic contents are released ${ }^{22}$. Both the rapidity of cell death and the morphological changes are hallmarks that distinguish pyroptotic from apoptotic cell death ${ }^{3}$. In some circumstances, GSDM-mediated cell membrane damage can be repaired by the ubiquitous damaged cell membrane repair response - the cell survives but inflammatory mediators are released through GSDM pores before the pores are removed from the membrane ${ }^{23}$. Macrophages that survive despite GSDMD cleavage have been termed 'hyperactivated', because they both stimulate inflammation and preserve their other antigen-presenting functions ${ }^{24}$.

The diverse physiological and pathological roles of GSDMs as executioners of pyroptosis are still being uncovered. However, associations of GSDMs with several diseases have emerged. GSDMD has been implicated in the pathogenesis of many inflammasome-associated diseases and tissue damage ${ }^{14,25,26}$. In addition, spontaneous mutations of GSDMs lead to disorders such as alopecia (GSDMA) $)^{27-31}$, asthma (GSDMB) ${ }^{32}$ and hear-

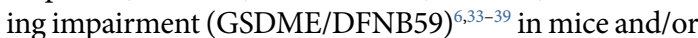
humans. Moreover, accumulating evidence suggests that GSDMs can inhibit or promote infection and cancers, implying complicated links between inflammation and pyroptosis and the development and progression of those diseases ${ }^{18-20,40-47}$.

The key roles of GSDMs in infection, inflammation and cancer have stimulated investigation of GSDMtargeting therapeutics. Strategies to therapeutically modulate GSDM activity are beginning to emerge, and several inhibitors of GSDMD have now been reported ${ }^{45,48,49}$.

\section{Box 1 | Discovery of pyroptosis}

Pyroptosis, a programmed, inflammatory cell death, is mediated by $\mathrm{N}$-terminal (NT) gasdermin pores (FIG. 2). Pyroptotic cells lose cell membrane integrity, which leads to release of inflammatory cellular contents and formation of large ballooning bubbles, which are much larger than apoptotic blebs ${ }^{22,186}$. The dying cell appears to flatten as its cytoplasmic contents are released.

The first descriptions of pyroptosis were in the 1990s in macrophages that died after infection with invasive Shigella flexneri or Salmonella enterica subsp. Typhimurium. At that time, invasive bacteria-induced cell destruction was regarded as apoptosis, as markers of apoptosis (DNA fragmentation and membrane blebbing) were observed ${ }^{187-190}$. Further studies showed that macrophage death in response to bacterial infection was mediated by inflammatory caspase 1 rather than apoptotic caspases and was associated with pro-inflammatory cytokine secretion ${ }^{190-195}$. In 2001, Cookson and Brennan coined the term pyroptosis (fiery death) to distinguish inflammatory caspase-mediated cell death from apoptosis and unprogrammed necrosis caused by lack of membrane integrity ${ }^{196}$. Gasdermin D (GSDMD) was identified in 2015 as the inflammatory caspase substrate responsible for pyroptotic cell death in immune cells, and the following year the mechanism responsible for GSDMD-mediated death was identified as pore formation by the inflammatory caspase-generated GSDMD-NT ${ }^{8-11,13,14,22}$. Identifying the molecular basis of pyroptosis has prompted an explosion of research to understand the role of pyroptosis in disease.
This Review encapsulates our current understanding of the expression, activation and regulation of GSDMs and highlights their important physiological and pathological roles as perpetrators of cell death, cytokine secretion and inflammation. Emerging opportunities to develop GSDM-targeted drugs, particularly GSDMD inhibitors, and the associated challenges, are discussed.

\section{The gasdermin family}

Humans have six GSDM genes: GSDMA, GSDMB, GSDMC, GSDMD, GSDME and DFNB59 (TABLE 1; FIC. 1). GSDME on human chromosome $7 \mathrm{p} 15.3$ is the most ancient gene with orthologues found in early metazoa and invertebrates ${ }^{17,50,51}$. The other GSDM genes likely arose as gene duplications - GSDMA and GSDMB on human chromosome 17q21 and GSDMC and GSDMD on human chromosome $8 \mathrm{q} 2$. Although in metazoa DFNB59 is not truncated and is closely homologous to GSDME, mammalian DFNB59 is a more distant and truncated gene, whose $\mathrm{N}$ terminus is homologous to the NT, pore-forming region of the other family members, but whether it possesses pore-forming activity is unknown. Mice lack a GSDMB orthologue, but have one Gsdmd on chromosome 15, one Gsdme on chromosome 6 and three Gsdmas (Gsdma1-3) and four Gsdmcs (Gsdmc1-4) clustered on chromosomes 11D and 15D1, respectively ${ }^{1}$.

Although published studies and web-based gene expression atlases are available for GSDM expression, these resources are inadequate for accurately predicting GSDM expression, which can be induced by cytokines and immune signalling in cells that are not ordinarily thought to express GSDMs, including lymphocytes. For example, GSDMA is upregulated by TGF $\beta^{52}$, GSDMB by interferon- $\alpha$ (IFN $\alpha$ ), IFN $\beta$, IFN $\gamma$ and TNF $^{19}$, GSDMD by IRF2 (REF. ${ }^{53}$ ) and GSDME by corticosteroids and forskolin ${ }^{54}$. Furthermore, expression of some of the GSDMs (especially of GSDMA and GSDME in cancer cell lines) is inferred to be epigenetically suppressed by DNA methylation, as DNA methyltransferase inhibitors can induce their expression ${ }^{55-60}$. Perhaps because of their expression in barrier cells, mutations in GSDM genes have been associated with skin defects, asthma and deafness in mice and humans (TABLE 2).

GSDMA. The first GSDM family member was identified by positional cloning of a gene causing abnormal skin and hair development in the mouse Rim 3 mutant ${ }^{5,61}$. Subsequent studies of mice with similar skin phenotypes $^{27-31}$ and bioinformatics ${ }^{62}$ identified a Gsdma1, Gsdma2 and Gsdma3 gene cluster on mouse chromosome $11 \mathrm{D}$ and found that Gsdma3 mutations caused alopecia (TABLE 2; FIG. 1). All these mutations localize to the CT domain of Gsdma3 and behave as gain-of-function mutations that unmask the functional NT domain ${ }^{9}$. Gsdma2 and Gsdma 3 are primarily expressed in stomach and skin, respectively ${ }^{27,52}$. The human GSDMA orthologue is also expressed in stomach and skin but is silenced in gastric cancer tissues and cell lines ${ }^{5}$ (TABLE 1). The DNA methyltransferase inhibitor 5-aza-2'-deoxycytidine (5-aza-dC) upregulates GSDMA in these cancer lines, suggesting that GSDMA expression is suppressed by DNA methylation ${ }^{55}$. In pit cells of the gastric epithelium, TGF $\beta$ 


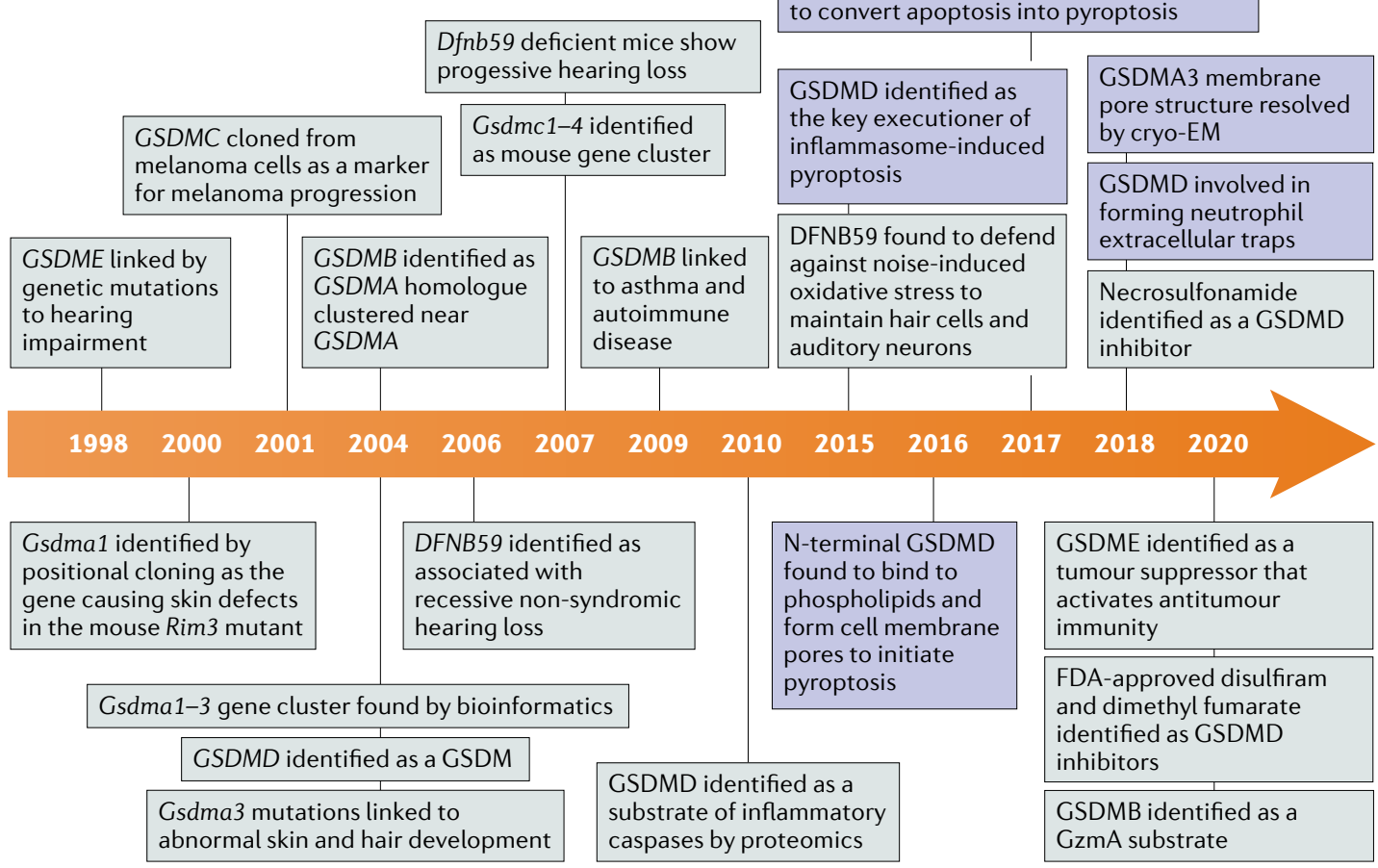

Fig. 1 Key events in the history of gasdermins. The purple boxes indicate milestone discoveries of gasdermin (GSDM) research.

induces the LIM domain only 1 (LMO1) transcription factor to upregulate GSDMA and trigger pit cell death ${ }^{52}$. TNF similarly upregulates Gsdma3 and causes death of mouse skin keratinocytes in vitro and in vivo ${ }^{63}$. It is worth noting that no obvious phenotype was observed in $\mathrm{Gsdma}^{-/-}$or $\mathrm{Gs} d \mathrm{ma}^{-/-}$mice $\mathrm{e}^{64,65}$, indicating that the Gsdma genes may be redundant in development and in the maintenance of gastric and skin barriers, or that their function is only apparent upon challenge by pathogens or other danger signals, which would be consistent with cleavage-dependent activation. GSDMA mutations have not been reported in human alopecia; however, GSDMA polymorphisms have been linked to systemic sclerosis (an autoimmune fibrotic disease of the skin and visceral organs), inflammatory bowel disease (IBD) and childhood asthma ${ }^{66-69}$ (TABLE 2).

Serine proteases stored in the cytotoxic granules of killer lymphocytes that are released during killer cell attack to cause programmed cell death in the target cell.

Pro-inflammatory cytokines Small molecular weight proteins secreted by immune cells and some other cells that evoke inflammatory responses, activate immune cells and cause inflammation, fever and tissue damage.

\section{Alarmins}

Host molecules released from damaged or dying cells that act as danger signals to stimulate inflammation, also known as damage-associated molecular patterns (DAMPs) GSDMB. The human GSDMB gene clusters with $G S D M A^{62}$, but no Gsdmb has been identified in rodents. $G S D M B$ is expressed more broadly than GSMDA, especially in airway and gastrointestinal epithelia, liver, and neuroendocrine and immune cells ${ }^{32,70-72}$ (TABLE 1). There are four GSDMB isoforms (1-4; Ensembl: ENSG00000073605), but whether these isoforms are expressed in a tissue or cell-specific manner is unknown. ered a strong association between GSDMB and susceptibility to chronic inflammatory diseases including IBD, asthma and type I diabetes ${ }^{32,73-76}$, and a single nucleotide polymorphism linked to increased GSDMB expression is associated with asthma ${ }^{32}$ (TABLE 2). The link to childhood asthma and IBD is shared between GSDMA and $G S D M B^{66,69,77}$, likely reflecting the close chromosomal locations of these two genes and their potentially coupled expression.

GSDMB isoform 1 is the dominant isoform in human bronchial epithelial cells, and elevated isoform 1 expression is found in patients with asthma compared with healthy controls ${ }^{32}$. GSDMB isoform 1 overexpression increases the levels of genes involved in airway responsiveness and remodelling including the genes encoding TGF $\beta 1$, 5-lipoxygenase and MMP9, which could explain its role in asthma ${ }^{32}$. Surprisingly, unlike other GSDMs, GSDMB isoform 1 was found exclusively in the nucleus, consistent with a postulated role in amplification of transcription ${ }^{32}$, but making it uncertain whether GSMDB-mediated pyroptosis is involved in the pathogenesis of asthma or IBD. More in vitro and in vivo evidence is required to understand whether the effects of GSDMB depend on pore formation.

Unlike GSDMA and GSDME, GSDMB expression is Genome-wide association studies (GWAS) have uncov- markedly increased in various cancers, including cervical, breast, gastrointestinal and hepatic cancers, and its high expression is associated with poor prognosis $\mathrm{s}^{71,78-80}$ (TABLE 1). However, whether different isoforms of GSDMB are expressed in distinct cancers or how GSDMB affects tumour biology is not known. The novel nuclear localiits function may be largely independent of pore-forming activity and raise the possibility that other GSDMs might also have unsuspected functions that are independent of their cleavage or ability to form pores.

GSDMC. GSDMC was first cloned from melanoma cells as a marker for melanoma progression ${ }^{81,82}$, and the mouse cluster (Gsdmc1-4) was identified by phylogenetic zation and transcriptional effect of GSDMB ${ }^{32}$ suggest that 
analysis ${ }^{1}$. GSDMC is expressed in trachea, spleen, oesophagus, small intestine, caecum, colon ${ }^{1,70,82}$ and in skin keratinocytes, where expression is markedly increased by UV radiation via calcium-triggered NFATc1 signalling ${ }^{83,84}$. GSDMC expression can also be induced in tumour cells
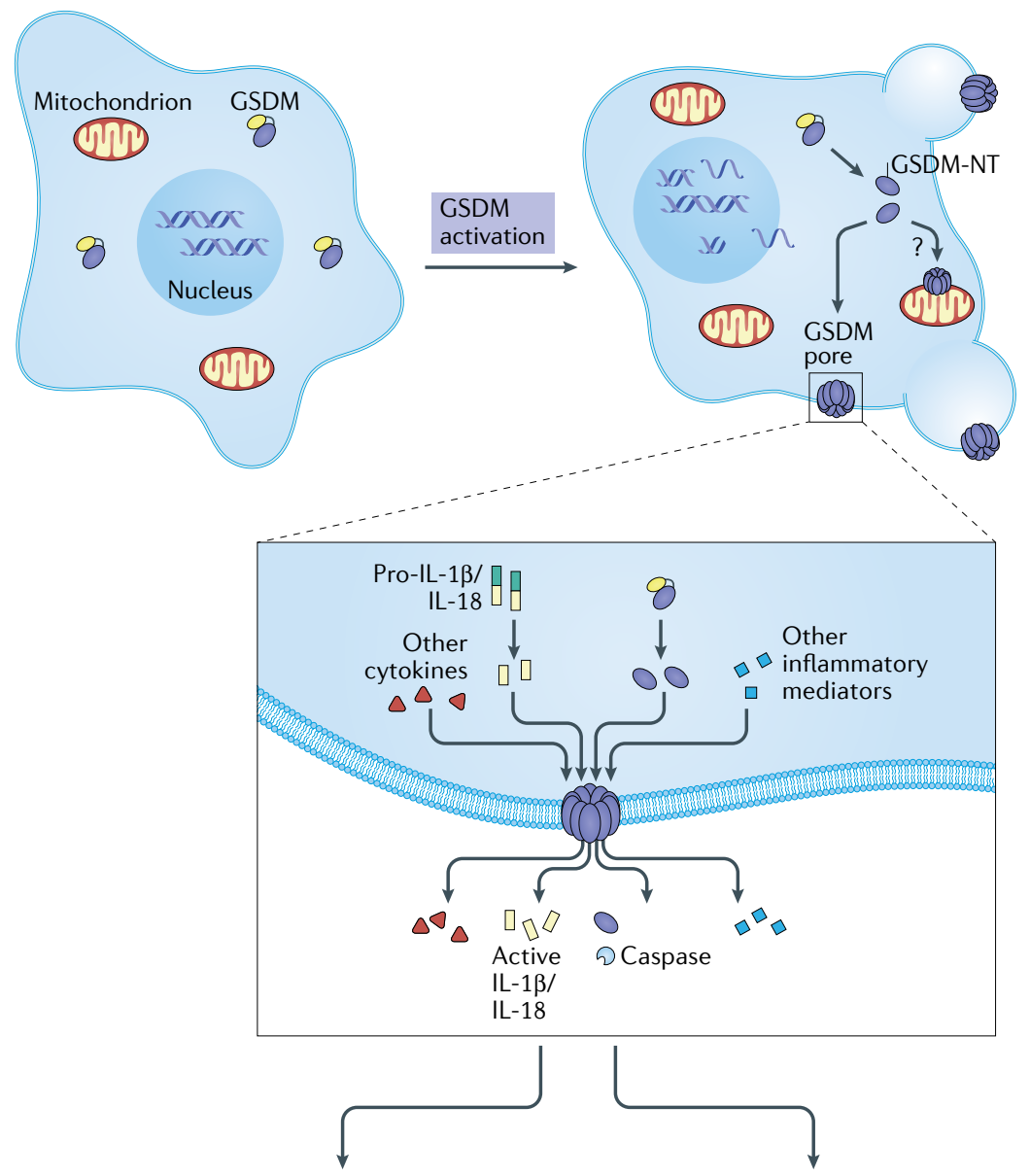

Hyperactivation
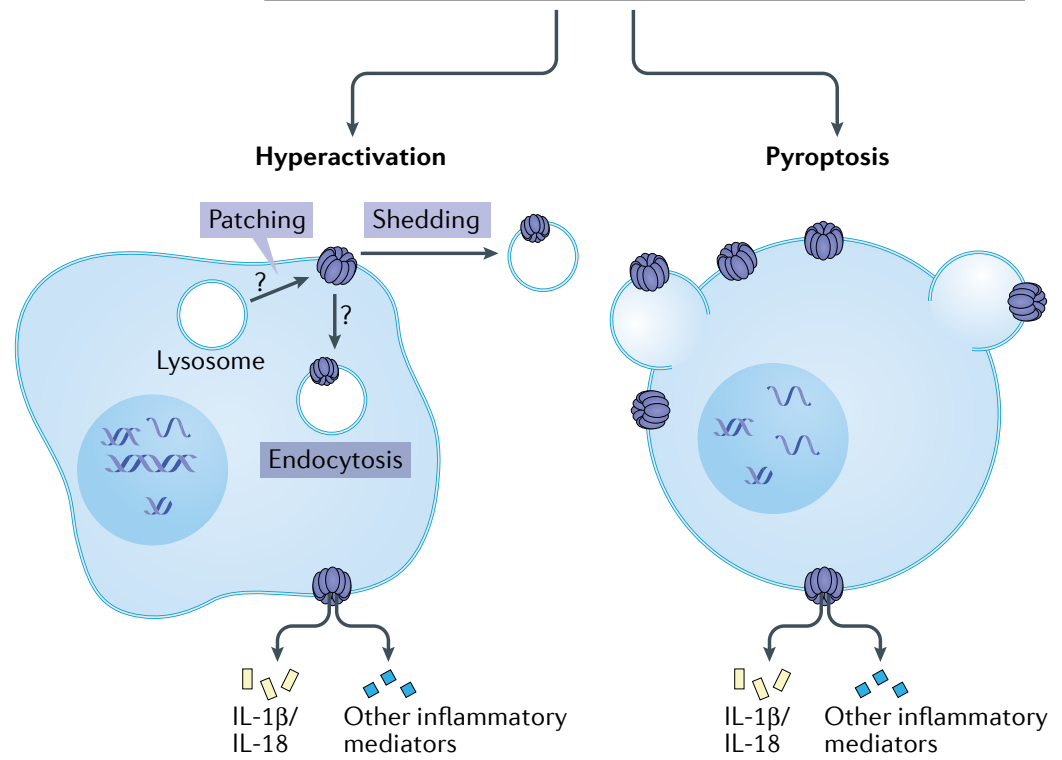

Fig. 2 | Gasdermins function as gatekeepers of pyroptosis. In response to invasive pathogens, sterile danger signals or cytotoxic T cell attack, gasdermins (GSDMs) are activated by proteolytic cleavage, which releases the $\mathrm{N}$-terminal (NT) fragment, which forms large cell membrane pores. The GSDM pore behaves as a gatekeeper for initiating downstream inflammatory cascades and pyroptotic cell death. Pyroptotic cells form large balloon-like membrane structures. Small intracellular molecules, including cytokines and cellular alarmins, are released through GSDM pores, causing inflammation. Some cells, termed 'hyperactivated', repair GSDM pores by shedding the damaged membrane and survive, but still induce inflammation by releasing IL-1 family cytokines. by hypoxia-triggered STAT3 phosphorylation and its complexation with nuclear-translocated PDL1 (REF. ${ }^{20}$ ) (TABLE 1). GSDMC contributes to UV-induced MMP1 expression by activating ERK and JNK pathways ${ }^{84}$. GSDMC is cleaved and activated by caspase 8 in death receptor signalling, converting apoptosis into pyroptosis in cells that express GSDMC ${ }^{20}$. GSDMC is also upregulated in colorectal cancer and promotes cancer cell proliferation $^{85}$, suggesting that GSDMC may function as an oncogene.

GSDMD. GSDMD was originally identified by homology to other GSDM genes on chromosome 8 near $G S D M C^{81}$. A 2010 proteomics study ${ }^{86}$ identified GSDMD as the most efficient (and also selective) substrate of inflammatory caspases. However, the functional role of GSDMD and its cleavage were not explored further until the discovery of GSDMD as the key executor of inflammasome-induced pyroptosis reported by two groups in 2015, using a chemical mutagenesis screen in mice and a cell-based CRISPR screen ${ }^{13,14}$.

GSDMD is expressed in immune cells (especially macrophages and dendritic cells) ${ }^{3}$, placenta, gastrointestinal epithelium, various cancers including oesophageal and gastric cancers, pancreatic cancer, prostate cancer, melanoma and salivary gland tumour, and Jurkat $\mathrm{T}$ and Ramos B cancer cell lines ${ }^{70,81,87}$ (TABLE 1). Transcription of GSDMD is upregulated by IRF2 $\left(\mathrm{REF}^{53}\right)$, which is a transcriptional suppressor of interferons. Although human GSDMD genetic polymorphisms have been identified, including a few that disrupt cleavage or pore formation ${ }^{88}$, most single nucleotide polymorphisms did not alter pore formation, and so far none has been linked to disease. The lack of known disease links does not mean that none exists, especially as the identification of GSDMD as the mediator of pyroptosis is so recent. Although GSDMD is widely expressed, no overt phenotype has so far been described in Gsdmd $d^{-/-}$mice, suggesting that GSDMD is largely dispensable for development and physiological homeostasis ${ }^{87}$. However, a recent study showed that the AIM2 cytosolic DNA-sensing inflammasome purges genetically compromised neuronal cells during neurodevelopment through GSDMD activation, preventing the accumulation of genetically aberrant cells and contributing to CNS homeostasis ${ }^{7}$. $\mathrm{Gsdmd}^{-/-}$mice, like Aim2 $2^{-/-}$, Asc $\mathrm{s}^{-/-}$or Casp $1^{-/-}$mice, exhibited increased anxiety in behavioural tests ${ }^{7}$. In models of infection, Gsdmd $d^{-/-}$mice can be more or less susceptible to infection than wild-type mice, depending on the pathogen ${ }^{40,44,89,90}$. However, Gsdmd $d^{-/-}$mice are protected from death and morbidity in many models of genetic or induced inflammation, including sepsis, experimental autoimmune encephalomyelitis (EAE, a mouse model for multiple sclerosis (MS)), macular degeneration and neonatal onset multisystem inflammatory disease (NOMID, caused by gain-of-function mutation of the inflammasome gene $N L R P 3)^{14,25,26}$, highlighting the central role of GSDMD in inflammatory disease.

GSDME. GSDME was first described as a mutated gene (DFNA5) that results in an autosomal dominant form of hearing impairment, caused by cochlear hair 
Table 1 | Human and mouse gasdermin genes

\begin{tabular}{|c|c|c|c|c|c|c|c|}
\hline $\begin{array}{l}\text { Human gene } \\
\text { (chromosomal } \\
\text { location) }\end{array}$ & $\begin{array}{l}\text { Mouse gene } \\
\text { (chromosomal } \\
\text { location) }\end{array}$ & $\begin{array}{l}\text { Expression in } \\
\text { normal tissues }\end{array}$ & $\begin{array}{l}\text { Expression in } \\
\text { tumours }\end{array}$ & $\begin{array}{l}\text { Activation } \\
\text { pathway }\end{array}$ & $\begin{array}{l}\text { Biological } \\
\text { function }\end{array}$ & Disease links & Refs \\
\hline $\begin{array}{l}\text { GSDMA } \\
(17 q 21.1)\end{array}$ & $\begin{array}{l}\text { Gsdma1-3 } \\
\text { (11D) }\end{array}$ & $\begin{array}{l}\text { Gastric and skin } \\
\text { epithelia }\end{array}$ & $\begin{array}{l}\text { Silenced in gastric } \\
\text { cancer tissues and } \\
\text { cell lines }\end{array}$ & Not known & Not known & $\begin{array}{l}\text { Systemic sclerosis } \\
\text { in humans, alopecia in } \\
\text { mice }\end{array}$ & $1,27,28,30,31,61$ \\
\hline $\begin{array}{l}\text { GSDMB } \\
(17 q 21.1)\end{array}$ & None & $\begin{array}{l}\text { Airway, } \\
\text { oesophagus, } \\
\text { gastrointestinal } \\
\text { tract, liver and } \\
\text { colon epithelium, } \\
\text { neuroendocrine } \\
\text { cells, immune cells }\end{array}$ & $\begin{array}{l}\text { Expressed in colon, } \\
\text { rectal, pancreatic } \\
\text { and cervical } \\
\text { cancers, and } \\
\text { barely expressed } \\
\text { in breast, lung and } \\
\text { liver cancers }\end{array}$ & Granzyme A & Not known & $\begin{array}{l}\text { Inflammatory bowel } \\
\text { disease, asthma, type I } \\
\text { diabetes }\end{array}$ & $19,32,72,74-80$ \\
\hline $\begin{array}{l}\text { GSDMC } \\
(8 \mathrm{q} 24.21)\end{array}$ & $\begin{array}{l}\text { Gsdmc1-4 } \\
\text { (15D1) }\end{array}$ & $\begin{array}{l}\text { Keratinocytes, } \\
\text { trachea, spleen, } \\
\text { oesophagus, small } \\
\text { intestine, caecum, } \\
\text { and colon }\end{array}$ & $\begin{array}{l}\text { Upregulated in } \\
\text { colorectal cancer } \\
\text { and melanoma }\end{array}$ & TNFR-caspase 8 & Not known & Not known & $20,82-85$ \\
\hline $\begin{array}{l}\text { GSDME } \\
\text { (7p15.3) (a.k.a. } \\
\text { DFNA5) }\end{array}$ & $\begin{array}{l}\text { Gsdme (6B2.3) } \\
\text { (a.k.a.Dfna5) }\end{array}$ & $\begin{array}{l}\text { Cochlea, placenta, } \\
\text { heart, brain, kidney }\end{array}$ & $\begin{array}{l}\text { Epigenetically } \\
\text { inactivated by } \\
\text { DNA methylation } \\
\text { in gastric, } \\
\text { colorectal and } \\
\text { breast cancer, } \\
\text { and most human } \\
\text { cancer cell lines }\end{array}$ & $\begin{array}{l}\text { Granzyme B, } \\
\text { caspase } 3\end{array}$ & Pyroptosis & $\begin{array}{l}\text { Autosomal dominant } \\
\text { nonsyndromic hearing } \\
\text { loss }\end{array}$ & $16-18,33-38$ \\
\hline
\end{tabular}

a.k.a., also known as.

\section{AIM2}

(Absent in melanoma 2).

An inflammasome that senses microbial or mislocalized host DNA in the cytosol to form a canonical inflammasome.

Experimental autoimmune encephalomyelitis

(EAE). Animal models of autoinflammatory disease in the central nervous system that resemble human multiple sclerosis, which are triggered by injecting immunogenic self peptides that sensitize $\mathrm{CD} 4^{+} \mathrm{T}$ cells.

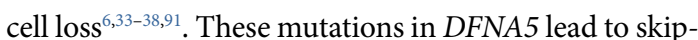
ping of exon 8, premature termination and activation of GSDME to cause cell death. GSDME mRNA is detected in cochlea, placenta, heart, brain and kidney ${ }^{6,92}$ (TABLE 1). GSDME is cleaved and activated by caspase 3 (and possibly other apoptotic effector caspases, caspases 6 and 7) when cells expressing GSDME are triggered to undergo apoptosis. GSDME cleavage converts noninflammatory apoptosis into inflammatory pyroptosis. Why GSDME-activating mutations specifically cause auditory damage, with no apparent effect on other organs in which it is highly expressed, is unclear. One possibility is that because the autoactive GSDME protein is unstable, pyroptosis could be limited to especially vulnerable cochlear hair cells ${ }^{17}$. The poor stability of autoactive GSDMs could also explain why there are no reports of disease-associated autoactive mutant GSDMs except for human GSDME and mouse GSDMA.

Relative to normal tissue, GSDME expression is epigenetically suppressed by methylation in gastric, colorectal and breast cancers, and most human cancer cell lines. By contrast, p53, which is inactivated in many cancers, reportedly activates GSDME transcription in DNA-damaged cells ${ }^{93}$, which could promote the removal of cancer cells by inflammatory recruitment and activation of phagocytic macrophages. GSDME is also mutated in a variety of other cancers, consistent with its postulated role as a tumour suppressor gene $\mathrm{e}^{56-60,94,95}$. When expression of GSDME is induced by DNA methyltransferase inhibitors in vitro, colony formation in gastric and colorectal cancers and invasiveness in breast cancer are reduced $^{56-59}$. Notably, cancer-associated GSDME mutations in the NT domain are primarily loss-of-function (LOF) mutations, which lead to impaired pore-forming ability $^{18}$ (TABLE 2).

DFNB59. DFNB59 is a more distantly related GSDM family member with a truncated non-homologous CT domain. DFNB59 transcripts are detected in brain, eye, inner ear, heart, lung, kidney, liver, intestine and testis ${ }^{39,96}$ (TABLE 1). Like GSDME, DFNB59 is linked by familial genetic mutations to hearing loss in humans, but these mutations are recessive, rather than dominant ${ }^{39}$. Patients with DFNB59 mutations show either dysfunctional cochlear outer hair cells ${ }^{97}$ or auditory neuropathy ${ }^{39}$, which impairs neural transmission of the auditory signal (TABLE 2). Mutations in mouse Dfnb59 were also found to be associated with deafness by forward genetic 
Neonatal onset multisystem inflammatory disease (NOMID). A rare severe autoinflammatory disease, caused by constitutively active mutations of the NLRP3 inflammasome gene, that occurs in infancy with symptoms of fever, chronic meningitis, skin rash and arthropathy, also known as chronic infantile neurologic cutaneous articular (CINCA) syndrome.

IL-1 family

A group of 11 pro-inflammatory

cytokines and their natural antagonists (IL-1 $\alpha, \mathrm{IL}-1 \beta$

IL-1RA, IL-18, IL-33, IL-36a, IL-36 $\beta$, IL-36 $\gamma$, IL-36RA,

IL-37 and IL-38) that share a conserved $\beta$-trefoil structure and have important roles in promoting or regulating inflammatory responses to infection or sterile insults.

\section{NLRP3}

A member of the NOD-like receptor subfamily of pattern recognition receptors, which recruits adaptor protein ASC and caspase 1 to assemble a large protein complex named the inflammasome in response to danger signals. Also known as NALP3 and cryopyrin

Pyrin

A cytosolic inflammasome sensor encoded by the MEFV gene, predominantly expressed in phagocytes, that is triggered by bacterial toxins or effectors. screening ${ }^{97}$. However, Dfnb59-/- mice, but not Gsdme ${ }^{-/-}$ mice, show hearing impairment ${ }^{39,97-99}$, suggesting that they cause deafness through different mechanisms.

DFNB59 is a peroxisome-associated protein required for oxidative stress-induced peroxisome proliferation in both hair cells and auditory neurons ${ }^{96}$, which protects against sound-induced oxidative damage. DFNB59 senses sound-induced reactive oxygen species (ROS) and recruits the autophagy machinery to degrade damaged peroxisomes ${ }^{100}$. It is unclear whether DFNB59 is a pore-forming protein or whether it is constitutively active, as its extremely short CT domain might not inhibit pore formation. It is worth exploring whether DFNB59 forms pores in peroxisome membranes and whether other interacting proteins inhibit or activate it. In support of the latter possibility, DFNB59-CT interacts with the ROCK2 Rho-associated protein kinase and the IQGAP1 scaffold protein $^{99}$.

\section{Role of gasdermins in pyroptosis}

The best-characterized pyroptosis pathway is that induced by GSDMD downstream of inflammasome activation in immune sentinel cells and some epithelial cells at mucosal and skin barriers (FIG. 3). Depending on the upstream inflammasome signals, the nature of the danger molecules released from dying cells may be different. Most canonical inflammasomes first require a transcriptional priming step (for example by lipopolysaccharide (LPS)-mediated Toll-like receptor signalling) to upregulate the expression of inflammasome sensors and pro-forms of IL-1 family cytokines ${ }^{101}$. When these canonical inflammasomes are activated, such as through the AIM2 sensor by cytosolic double-stranded DNA (dsDNA), the NLRP3 sensor by a large array of stimuli that disturb cell membrane integrity (for example bacterial toxin nigericin, extracellular ATP, uric acid crystals, cholesterol crystals and amyloid aggregates) or the pyrin sensor by the Clostridioides difficile toxin TcdB, IL-1 family cytokines IL- $1 \beta$ and IL- 18 are processed to maturity ${ }^{102,103}$ and GSDMD is cleaved at Asp275 (mouse Asp276) in the interdomain linker ${ }^{13,14}$. By contrast, noncanonical inflammasomes that activate caspases 4,5 and 11 proteolyse only GSDMD, but not IL-1 family cytokines even with priming; however, secondary GSDMD pore-induced membrane damage and NLRP3 activation result in cytokine maturation in addition to GSDMD processing ${ }^{14,104}$.

GSDMD pores in the plasma membrane trigger rapid pyroptotic cell death in which the damaged membrane forms large ballooning bubbles and dying cells appear to flatten as their cytoplasmic contents are released ${ }^{22}$ (FIG. 2). How or whether GSDMD pore-induced cell death is regulated is not clear, but cells with activated GSDMD pores do not always die. Both the rapidity of cell death and the morphological changes are hallmarks that easily distinguish pyroptotic from apoptotic cell death ${ }^{3}$. The cellular contents that are released from pyroptosis can include IL- 1 family cytokines IL- $1 \beta$ and IL-18, as well as IL-1 $1 \alpha$ that does not require processing for its biological activity. Of note, neither full-length nor processed IL-1 family cytokines have a signal peptide for secretion via the endoplasmic reticulum to Golgi secretory pathway, but pyroptosis provides a mechanism for their release by 'unconventional protein secretion'. Other released contents include cellular alarmins, such as ATP and HMGB1, cleaved GSDMD, inflammatory caspases, chemokines and other cytokines. These signals amplify inflammation in the tissue and recruit immune cells to respond to infection or danger. Many small molecules and other proteins, which may differ from cell type to cell type and from stimulus to stimulus, are probably released from pyroptotic cells. Detailed unbiased studies are required to elucidate what molecules are released under what condition and which released molecules are particularly important for amplifying inflammation.

In addition to inflammatory caspases, caspase 8, which is activated by cell surface death receptor ligation and oligomerization, can trigger GSDMD-dependent pyroptotic cell death (FIG. 3). During pathogenic Yersinia infection, TAK1 or IкB kinase inhibition by the Yersinia effector protein YopJ unleashes RIPK1caspase 8-dependent GSDMD cleavage, which contributes to host defence against Yersinia infection ${ }^{105-107}$. Meanwhile, GSDME also gets cleaved and activated by an unknown mechanism and functions in concert with active GSDMD to trigger pyroptosis of infected cells. Similarly, in intestinal epithelial cells that lack FADD, RIPK1 and RIPK3-mediated caspase 8 activation leads to GSDMD-dependent pyroptosis and causes ileitis ${ }^{108}$. Like FADD, cellular FLICE-like inhibitory protein (cFLIP) negatively regulates pyroptotic cell death and inflammation. Deficiency of cFLIP relieves the inhibition of LPS-induced formation of a death receptor complex, which contains RIPK1 and caspase 8, leading to caspase 8-dependent cleavage of GSDMD ${ }^{109}$.

Pyroptosis can be induced by other GSDMs that are not activated by inflammasomes and without involving the IL-1 family cytokines (FIG. 3). During classical apoptosis, caspase 3 activates GSDME by cleaving after Asp270 (mouse Asp271), which converts relatively slow noninflammatory apoptotic death into a more rapid inflammatory pyroptotic death in cells that express GSDME ${ }^{16,17}$. By contrast, activated caspase 3 cleaves GSDMD within the $\mathrm{N}$ terminus and destroys its ability to form pores ${ }^{110}$. $\mathrm{GzmB}$, which is a serine protease that, like caspases, cleaves after Asp residues, is released from killer lymphocytes into target cells and can also directly cleave cytosolic GSDME to trigger pyroptosis ${ }^{18}$. GzmB also cleaves and activates caspase 3 , which amplifies its effectiveness at killing target cells ${ }^{111-113}$. GzmA, another death-inducing protease in killer lymphocytes, cleaves GSDMB that is expressed in digestive tract epithelial tumours and causes pyroptotic cell death in target tumour cells ${ }^{19}$. Collectively, although immune-mediated elimination of infected or cancerous target cells has always been thought of as noninflammatory, killer cells cause inflammatory pyroptosis in target cells expressing GSDME or GSDMB. Furthermore, a recent study showed that GSDMC can also be cleaved by caspase 8 after TNF-mediated death receptor signalling to trigger pyroptosis ${ }^{20}$.

\section{Regulation of gasdermin activity}

Structural studies over the past few years have helped to reveal the elegant mechanisms that control and activate GSDMs, from auto-inhibition to processing, and 
GSDMC/E-expressing cells Apoptotic stimuli

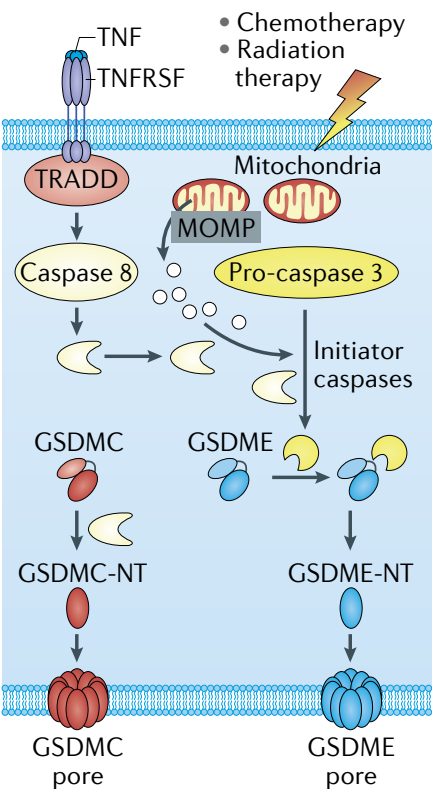

GSDMB/E-expressing target cells Killer cell attack

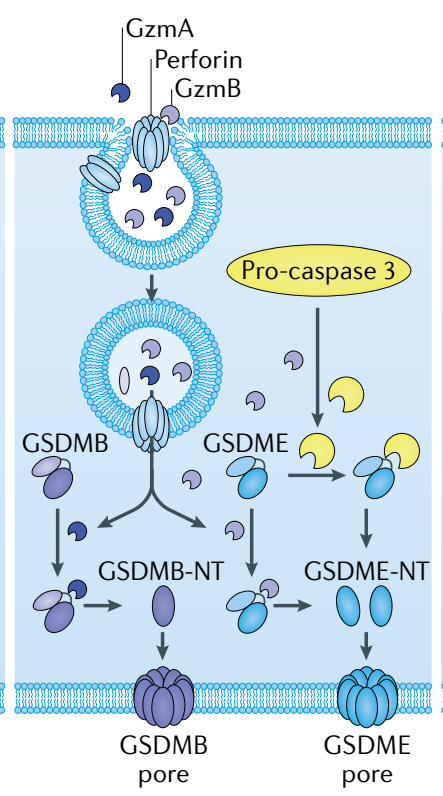

Immune cells

Inflammasome stimuli

DAMPs (ATP, PAMPs (LPS,

crystals) microbial toxins)

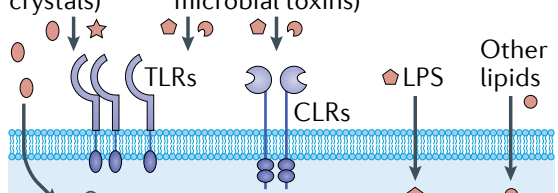

శ్
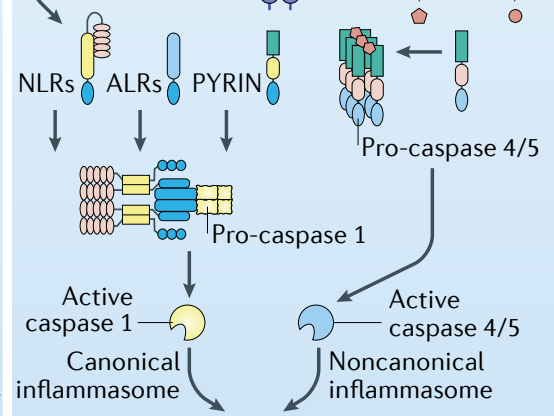
inflammasome

GSDMD
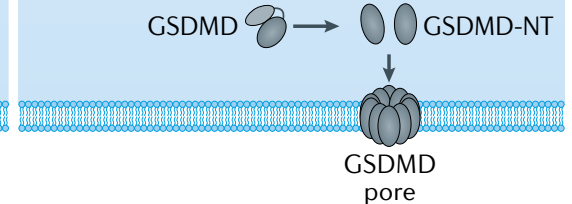

Active caspase $8 \quad$ Active caspase $3 \quad$ OCytochrome $c$

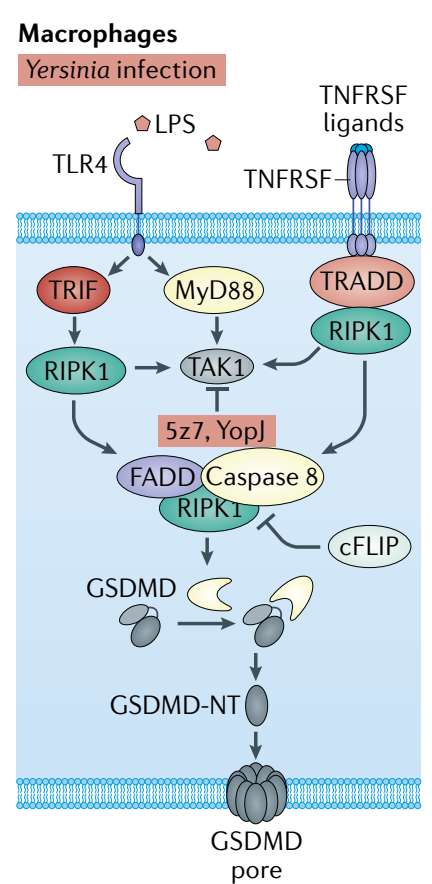

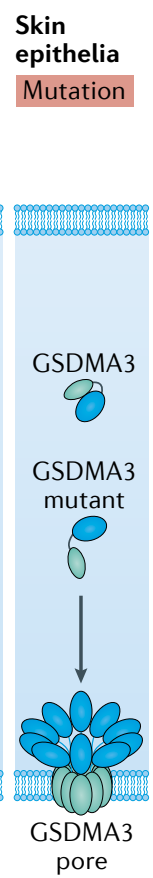

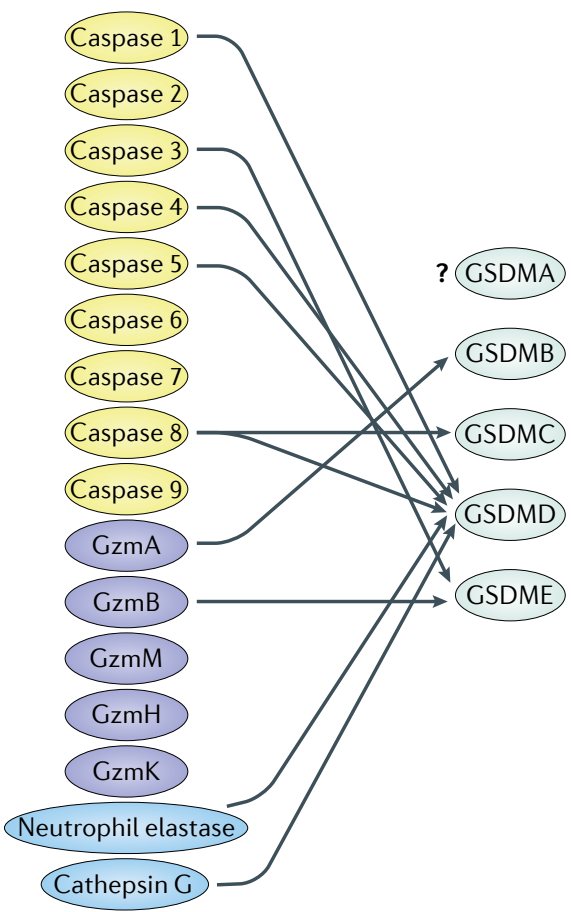

finally to formation of large $\beta$-barrel pores (FIG. 4a). The molecular basis of GSDM auto-inhibition has been illustrated by crystal structures of full-length, inactive mouse GSDMA3 and human and mouse GSDMD ${ }^{9,12}$. Whereas GSDMA3 was readily crystallized ${ }^{9}$, extensive loop truncations were required to crystallize either human or mouse GSDMD ${ }^{12}$. In both GSDMA3 and GSDMD full-length structures, the pore-forming NT

segments of the proteins comprise a twisted $\beta$-sheet surrounded by $\alpha$-helices, and a large part of the polypeptide chain that inserts as extended $\beta$-strands into the membrane is disordered. The corresponding GSDM-CTs, the repressive segment of the proteins, are compact and almost exclusively a-helical and interact extensively with the GSDM-NTs to inhibit pore formation. 
4 Fig. 3 | Molecular mechanisms that activate gasdermins. In response to apoptotic signalling, gasdermin C (GSDMC) and GSDME are processed by caspase 8 and caspase 3 , respectively, converting apoptosis into pyroptosis. Granzymes (Gzms) secreted by killer cells are delivered by perforin into target cells, where GzmA and GzmB can directly cleave and activate GSDMB and GSDME, respectively, to trigger pyroptotic cell death. In immune sentinel cells, both cytosolic canonical inflammasomes that respond to microbial infection or danger signals and the noncanonical inflammasome that responds directly to lipopolysaccharide (LPS) or endogenous oxidized phospholipids activate the inflammatory caspases (caspases 1, 4, 5 and 11), which cleave GSDMD and generate pore-forming $\mathrm{N}$-terminal GSDMD (GSDMD-NT). TAK1 inhibition by the Yersinia effector protein YopJ or the small molecule $5 z 7$ triggers caspase 8-dependent GSDMD cleavage and activation. GSDMD can also be directly processed and activated by neutrophil elastase (ELANE) and cathepsin G. In addition to protease-mediated release of active GSDM-NT, mutations in Gsdma3 lead to abolition of C-terminal GSDM inhibition and trigger GSMDA3 pore-forming activity. Diagram at bottom right indicates the proteases known to cleave and activate each of the gassdermins (yellow, caspases; purple, lymphocyte granzymes; blue, myeloid cell granule proteases). ALR, AIM-2 like receptor; CLR, C type lectin receptor; DAMP, damage-associated molecular pattern; NLR, NOD-like receptor; MOMP, mitochondrial outer membrane permeabilization; PAMP, pathogen-associated molecular pattern; TLR, Toll-like receptor.

Some mutations, especially in the $\mathrm{C}$ terminus of GSDMs, that disrupt the inhibitory interaction between the CT and NT fragments activate GSDMs spontaneously (TABLE 2). Both spontaneous and mutagenesis-induced Gsdma3 mutants acquire pore-forming activity and induce inflammatory cell death, which leads to mouse skin abnormalities ${ }^{27-31,61}$. GSDME mutations that cause familial hearing loss result in a truncated GSDME with spontaneous pore-forming activity ${ }^{6,33-38}$. The pores formed by these mutants are believed to be constructed with the dissociated $\mathrm{C}$ terminus still attached, although this has not been formally verified.

Based on the GSDMA3 structure, there are two auto-inhibitory GSDM NT-CT interfaces. Interface I is located near the centre of the protein and interface II at the periphery (FIG. 4b). The biophysical determinants of these two interfaces differ remarkably. Interface I, formed between the positively charged $\alpha 1$ helix and $\beta 1-\beta 2$ loop of GSDM-NT and an acidic groove of GSDM-CT, is primarily mediated by charge-charge interactions. By contrast, interface II is established through mainly hydrophobic interactions between the a 4 helix of GSDM-NT and GSDM-CT. Gain-of-function mutations in GSDM-associated diseases disrupt either of the auto-inhibitory contacts (TABLE 2), bypassing the requirement of proteolysis in GSDM activation and leading to membrane penetration by full-length $\mathrm{GSDMs}^{9,12}$.

Enzymatic processing of GSDMD for its activation (FIG. 4a) is more complex than classical substrate engagement by inflammatory caspases. The crystal structure of active caspase 1 in complex with a GSDMD interdomain linker fragment suggested that the catalytic pocket of inflammatory caspases recognizes the FLTD tetrapeptide motif (residues 272-275 in human GSDMD) ${ }^{114}$. However, the crystal structures of active caspases 1/4/11 in complex with GSDMD-CT and further functional characterization revealed a conserved mechanism whereby these inflammatory caspases interact with GSDMD-CT, instead of the tetrapeptide motif in the linker region, to drive the recognition and processing of GSDMD ${ }^{115}$ (FIG. 4b). The GSDMD mutant with FLTD mutated to AAAD was equally processed in vitro and in cells, and rescued GSDMD deficiency in LPS-induced pyroptosis $^{115}$. In the structures of the complex between caspase $1 / 4 / 11$ and GSDMD-CT, the autoprocessed inflammatory caspase forms a two- $\beta$-strand protrusion that acts as a substrate-binding exosite to recruit GSDMD by hydrophobic and hydrogen bonding interactions with the CT. This recruitment likely places the interdomain linker near the caspase catalytic site for cleavage, a hypothesis now supported by the crystal structure of full-length GSDMD in complex with caspase 1 (REF. ${ }^{116}$ ) (FIG. 4b). Thus, GSDMD-CT not only auto-inhibits GSDMD-NT, it also provides the platform for inflammatory caspase recruitment and GSDMD activation. It remains to be seen whether similar mechanisms regulate activation of other GSDMs.

\section{Mechanism of gasdermin pore formation}

The mechanism of GSDM pore formation was revealed from the structure of the GSDMA3-NT pore ${ }^{117}$. Because GSDM-NT pores are cytotoxic, preparing the GSDMA3-NT protein for structural studies involved expressing an engineered recombinant full-length GSDMA3 in which a human rhinovirus $3 \mathrm{C}$ protease site was inserted in the interdomain linker. The recombinant purified protein was then cleaved by the $3 \mathrm{C}$ protease in the presence of liposomes containing the acidic lipid cardiolipin. GSDMA3-NT pores formed on the liposomes were then solubilized in detergent and imaged by cryo-electron microscopy (cryo-EM). The highresolution structure is a strikingly large, predominantly 27 -subunit transmembrane $\beta$-barrel with 108 extended $\beta$-strands and an inner diameter of $18 \mathrm{~nm}$ (FIG. 4b). This observed dimension for GSDMA3-NT pores is on a par with the $20 \mathrm{~nm}$ ring dimension for GSDMD-NT pores measured by atomic force microscopy ${ }^{10,118}$. GSDM pores are sufficiently large to allow the passage of small or medium-sized proteins, including the IL-1 family cytokines (FIG. 4b).

The membrane-inserted GSDMA3-NT subunit resembles a left hand with its soluble globular domain as the palm and two membrane-inserted $\beta$-hairpins as fingers (FIG. $4 \mathrm{C}$ ). Comparison of auto-inhibited with membrane-inserted GSDMA3-NT structures indicated drastic conformational changes that result in the extension of the fingers as if a closed fist unfurls to an open hand, including melting of the $\alpha 4$ helix at the tip of a $\beta$-hairpin ${ }^{9,117}$ (FIG. 4C). In close juxtaposition to the inserted $\beta$-strands is the positively charged $\alpha 1$ helix, which interacts with cardiolipin in the cryo-EM structure and serves as a major acidic lipid-binding element. This $\alpha 1$ helix looks like a thumb folded over the palm. The $\alpha 1$ and $\alpha 4$ (FIG. 4c) helices of GSDM-NT, which are important in lipid binding and membrane insertion, respectively, are masked in the full-length protein by interactions with GSDM-CT (FIG. 4b), which explains how GSDM-CT represses GSDM activity. Oligomerization in the GSDMA3-NT pore is mediated by the sides of the hand-shaped subunit, from the membrane-inserted fingers, folded thumb to the palm ${ }^{117}$. In particular, the $\alpha 1$ thumb helices in the oligomerized pore align head to tail on the membrane to form a stabilizing helical belt. 


\section{Box 2 | Overview of apoptosis}

Classical apoptosis, a physiological noninflammatory programme of cell suicide, is triggered by a variety of stimuli including death receptor signalling, cytotoxic drugs, radiation and a variety of cellular stresses (including growth factor deprivation and hypoxia) that lead to mitochondrial outer membrane permeabilization (MOMP) and the activation of apoptotic effector caspases (caspases 3, 6 and 7) ${ }^{197,198}$. During apoptosis, mitochondria generate rective oxygen species (ROS), lose their transmembrane potential and release cytochrome $c$ into the cytosol, cell membrane lipids become scrambled and externalize phosphatidyl serine, chromatin condenses, genomic DNA is fragmented, mRNA is globally degraded and new protein synthesis is blocked ${ }^{197,199-201}$. Phagocytic cells, which recognize cell surface phosphatidylserine on apoptotic cells, rapidly engulf and remove apoptotic cells in vivo before their plasma membrane loses its integrity 202,203 . Thus, apoptotic cells do not release their cellular contents into the extracellular milieu, and apoptotic death is usually noninflammatory and immunologically silent. Although pyroptosis and apoptosis have historically been considered separate pathways, recent studies discussed in this Review show that apoptotic stimuli can trigger pyroptosis under certain circumstances. For example, activated caspase 3 can cleave gasdermin E (GSDME) to cause pyroptosis instead of apoptosis ${ }^{16,17}$. Similarly, in cells infected with pathogenic Yersinia, TNF receptor activation activates caspase 8 to trigger GSDMD-dependent pyroptosis ${ }^{105,106}$.

Interestingly, phosphorylation of Thr6 of $\alpha 1$ in GSDME (equivalent to Thr8 in GSDMA3) inhibits oligomer formation and pyroptosis ${ }^{119}$, presumably by altering the $\alpha 1$ helix conformation to interfere with oligomerization. The kinases responsible for phosphorylating GSDME or other GSDMs and how phosphorylation is regulated are unknown.

\section{Gasdermin D-mediated cell hyperactivation}

In certain situations, macrophages, dendritic cells and neutrophils survive inflammasome-activated GSDMD cleavage without membrane rupture and pyroptosis, and are called hyperactivated cells because they both stimulate inflammation and preserve their other functions ${ }^{24,120}$ (FIG. 2). Based on the cryo-EM structure of the GSDMA3-NT pore with an $\sim 18 \mathrm{~nm}$ inner diameter ${ }^{117}$, GSDMD-NT pores are also big enough for chemicals (such as ATP) and small proteins to pass through ${ }^{9,117}$. Hyperactivation and pyroptosis can be distinguished by measuring cell culture supernatants for lactate dehydrogenase (LDH), which is too large to exit through GSDMD-NT pores and relies on cell lysis for its release, and for mature IL-1 family cytokines and possibly HMGB1, which are small enough to be readily released through these pores. While pyroptotic cells release both LDH and cytokines, hyperactivated cells do not release $\mathrm{LDH}$, but do release cytokines ${ }^{21}$. Because hyperactivated cells survive, they may even process and pump out more inflammatory mediators than cells that die. An unbiased proteomics analysis of molecules released from hyperactivated cells and an analysis of their roles in infection and inflammation will be informative.

A number of processes that activate live cell cytokine secretion or hyperactivation have been described. In dendritic cells, a mixture of oxidized phosphorylcholine derivatives (oxPAPC) or an isolated component 1-palmitoyl-2-glutaryl-sn-glycero-3-phosphocholine (PGPC) produced in dying mammalian cells induces noncanonical inflammasome-dependent release of IL- $1 \beta$ without causing cell death ${ }^{120}$. Multiple stimuli, including an $N$-acetylglucosamine fragment of bacterial peptidoglycan and oxPAPC, can also hyperactivate macrophages and induce inflammasome-mediated IL- $1 \beta$ release from living macrophages in a GSDMD pore-dependent process ${ }^{21,24,121,122}$. Human, but not murine, circulating monocytes can respond to extracellular LPS to activate an alternative NLRP3-ASC-caspase 1 inflammasome independently of $\mathrm{K}^{+}$efflux and without ASC speck formation, leading to IL- $1 \beta$ secretion without cell death ${ }^{123}$. A TLR4-TRIF-RIPK1-FADD-caspase 8 signalling axis was dissected genetically as upstream of alternative inflammasome activation ${ }^{123}$, but the exact mechanism remains unknown. A hyperactive state can also be brought about by deleting the sterile- and armadillo-motif-containing protein 1 (SARM1), which also contains a Toll-IL-1R (TIR) domain ${ }^{124}$. Sarm1 $1^{-/-}$ macrophages essentially recapitulate NLRP3-dependent hyperactivation after priming and nigericin treatment, with release of IL- $1 \beta$ but without cell death ${ }^{124}$. Potentiation of cell death by SARM1 involves SARM1 clustering at the mitochondria and SARM1-dependent mitochondrial depolarization ${ }^{124}$.

Interestingly, exposure to Salmonella enterica subsp. Typhimurium, ATP or nigericin, which cause inflammasome activation and pyroptosis in macrophages, induces GSDMD-dependent IL- $1 \beta$ release in neutrophils, without killing them ${ }^{122,125,126}$. With some stimuli, GSDMD-NT in neutrophils predominantly targets primary granules and $\mathrm{LC}^{+}$autophagosomes, instead of the plasma membrane, and IL- $1 \beta$ appears to be secreted via an autophagy-dependent mechanism ${ }^{127}$. The resistance of neutrophils to pyroptotic death is unique among inflammasome-signalling cells so far described. It allows neutrophils to secrete IL- $1 \beta$ at sites of infection without compromising the crucial inflammasome-independent neutrophil antimicrobial functions that would be impaired if neutrophils died. The inflammasome pathway must somehow be modified in neutrophils to optimize host pro-inflammatory and antimicrobial responses during pathogen challenge.

What determines whether GSDMD cleavage triggers pyroptosis or hyperactivation is not well understood. However, a likely mechanism responsible for cell survival in the face of membrane damage by GSDM pores is activation of the ubiquitous plasma membrane damage repair response in eukaryotic cells, which rapidly restores membrane integrity, allowing the cell to survive. All cells sense damage to the plasma membrane by an immediate increase in intracellular $\mathrm{Ca}^{2+}$, which triggers membrane repair by recruiting the endosomal sorting complexes required for transport (ESCRT) machinery to damaged membrane areas and removes them in ectosomes $^{23}$. The plasma membrane repair response to other types of membrane damage also involves increased endocytosis of damaged membrane and patching of damaged membrane by donated vesicular membranes (mostly from lysosomes) ${ }^{128}$. Whether these other membrane repair phenomena occur in cells with GSDMD-damaged plasma membranes has not been examined. The ability of cells to survive and repair the damage likely reflects a contest between how much and how rapidly membrane damage is induced and whether it is spatially localized, versus the robustness of the 
Table 2 | Disease-linked gasdermin mutations

\begin{tabular}{|c|c|c|c|c|c|}
\hline Gene $^{a}$ & Nucleotide change & Amino acid change & Consequence & Disease link & Refs \\
\hline \multirow[t]{2}{*}{ GSDMA } & $53 \mathrm{G}>\mathrm{A}$ & $\mathrm{R} 18 \mathrm{Q}$ & Unknown & $\begin{array}{l}\text { Asthma/systemic } \\
\text { sclerosis }\end{array}$ & 67 \\
\hline & $382 G>A$ & V128M & $\begin{array}{l}\text { Elevated IgE and bronchial } \\
\text { hyperresponsiveness }\end{array}$ & Asthma & 69 \\
\hline \multirow[t]{8}{*}{ Gsdma3 } & - & Lacks auto-inhibition interfaces I and II: & \multirow{8}{*}{$\begin{array}{l}\text { Disrupts auto-inhibition, and } \\
\text { Gsdma3 becomes constitutively } \\
\text { active to induce pyroptosis in } \\
\text { hair follicle cells }\end{array}$} & \multirow[t]{8}{*}{ Alopecia } & \multirow[t]{8}{*}{$9,27-31$} \\
\hline & B2 insertion & $1-259+$ RDW & & & \\
\hline & $832 \mathrm{~A}>\mathrm{C}$ & $\mathrm{T} 278 \mathrm{P}$ & & & \\
\hline & $1028 \mathrm{~T}>\mathrm{C}$ & L343P & & & \\
\hline & $1030 \mathrm{~T}>\mathrm{C}$ & $\mathrm{Y} 344 \mathrm{H}$ & & & \\
\hline & $1031 \mathrm{~A}>\mathrm{G}$ & Y344C & & & \\
\hline & - & Disrupts auto-inhibition interface II: & & & \\
\hline & 1231_1236dup & Duplication of E411A412 & & & \\
\hline \multirow[t]{4}{*}{ GSDMB } & $871 G>A ; 892 C>T$ & G291R; P298S & \multirow[t]{2}{*}{ Unknown } & \multirow[t]{4}{*}{ Asthma } & 133 \\
\hline & & $\begin{array}{l}\text { Reduce conformational flexibility and } \\
\text { increase positive surface charge }\end{array}$ & & & \\
\hline & $236-1199 \mathrm{G}>\mathrm{A}$ & Intron variant & $\begin{array}{l}\text { Elevated lgE and bronchial } \\
\text { hyperresponsiveness }\end{array}$ & & 69 \\
\hline & $661+742 T>C$ & Exon 6 (13 aa) deletion & Unknown & & 77 \\
\hline \multirow[t]{9}{*}{ GSDME } & $990+503$ & \multirow{2}{*}{$\begin{array}{l}\text { Exon } 8 \text { skipping and truncated GSDME } \\
\text { containing aa } 1-330 \text { and an aberrant } \\
\text { 41-residue C-terminal sequence due } \\
\text { to frameshift (lacks auto-inhibition } \\
\text { interfaces I and II) }\end{array}$} & \multirow{2}{*}{$\begin{array}{l}\text { Truncated GSDME is } \\
\text { constitutively active to induce } \\
\text { pyroptosis in cochlear hair cells }\end{array}$} & \multirow{2}{*}{$\begin{array}{l}\text { Autosomal dominant } \\
\text { hearing loss }\end{array}$} & \multirow[t]{2}{*}{ 6,17,33-38,92 } \\
\hline & 990+1691delins 132 & & & & \\
\hline & - & Disrupts oligomerization interface: & \multirow{7}{*}{$\begin{array}{l}\text { Most GSDME mutants have } \\
\text { reduced pore-forming activity } \\
\text { and may have reduced tumour } \\
\text { inhibition }\end{array}$} & \multirow{7}{*}{ Cancer } & \multirow{7}{*}{18} \\
\hline & $53 \mathrm{~A}>\mathrm{T}$ & D18V & & & \\
\hline & $70 A>G$ & N24D & & & \\
\hline & $635 \mathrm{C}>\mathrm{T}$ & $\mathrm{P} 212 \mathrm{~L}$ & & & \\
\hline & - & Lacks extension domains I and II: & & & \\
\hline & $138 \mathrm{G}>\mathrm{A}$ & W46* & & & \\
\hline & $628 \mathrm{G}>\mathrm{T}$ & $\mathrm{E} 210^{\star}$ & & & \\
\hline \multirow[t]{8}{*}{ DFNB59 } & 113dupT & $\mathrm{K} 41 \mathrm{Efs}^{\star} 8$ (48 aa truncation) & \multirow{8}{*}{$\begin{array}{l}\text { T54I and R183W mutants result } \\
\text { in auditory neuropathy while } \\
\text { other truncations lead to defect } \\
\text { in cochlear hair cell function }\end{array}$} & \multirow{8}{*}{$\begin{array}{l}\text { Autosomal recessive } \\
\text { hearing loss }\end{array}$} & \multirow[t]{8}{*}{$39,97-100$} \\
\hline & 122 delA & $\mathrm{K} 41 \mathrm{Sfs}{ }^{\star} 18$ & & & \\
\hline & $161 C>T$ & T54l & & & \\
\hline & $406 \mathrm{C}>\mathrm{T}$ & $\mathrm{R} 136^{*}$ & & & \\
\hline & $499 \mathrm{C}>\mathrm{T}$ & T167* & & & \\
\hline & $547 \mathrm{C}>\mathrm{T}$ & R183W & & & \\
\hline & 726delT & $\mathrm{F} 242 \mathrm{Lfs}^{*} 7$ & & & \\
\hline & 988delG & V330Lfs*7 & & & \\
\hline Dfnb59 & $868 \mathrm{~A}>\mathrm{T}$ & $\mathrm{K} 290^{*}$ & $\begin{array}{l}\text { Defect in cochlear hair cell } \\
\text { function }\end{array}$ & $\begin{array}{l}\text { Autosomal recessive } \\
\text { hearing loss }\end{array}$ & 98 \\
\hline
\end{tabular}



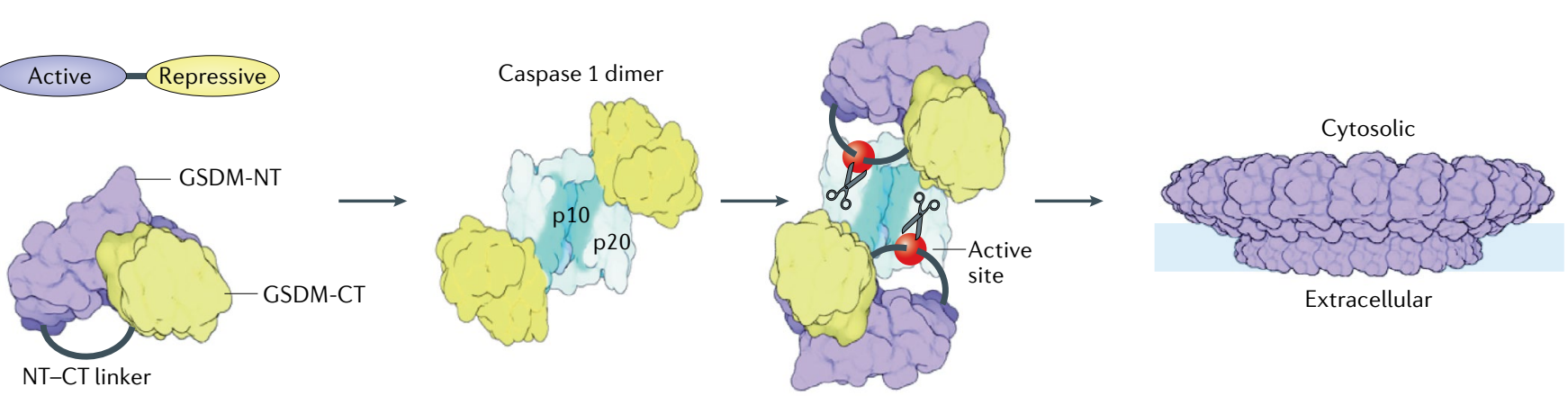

b
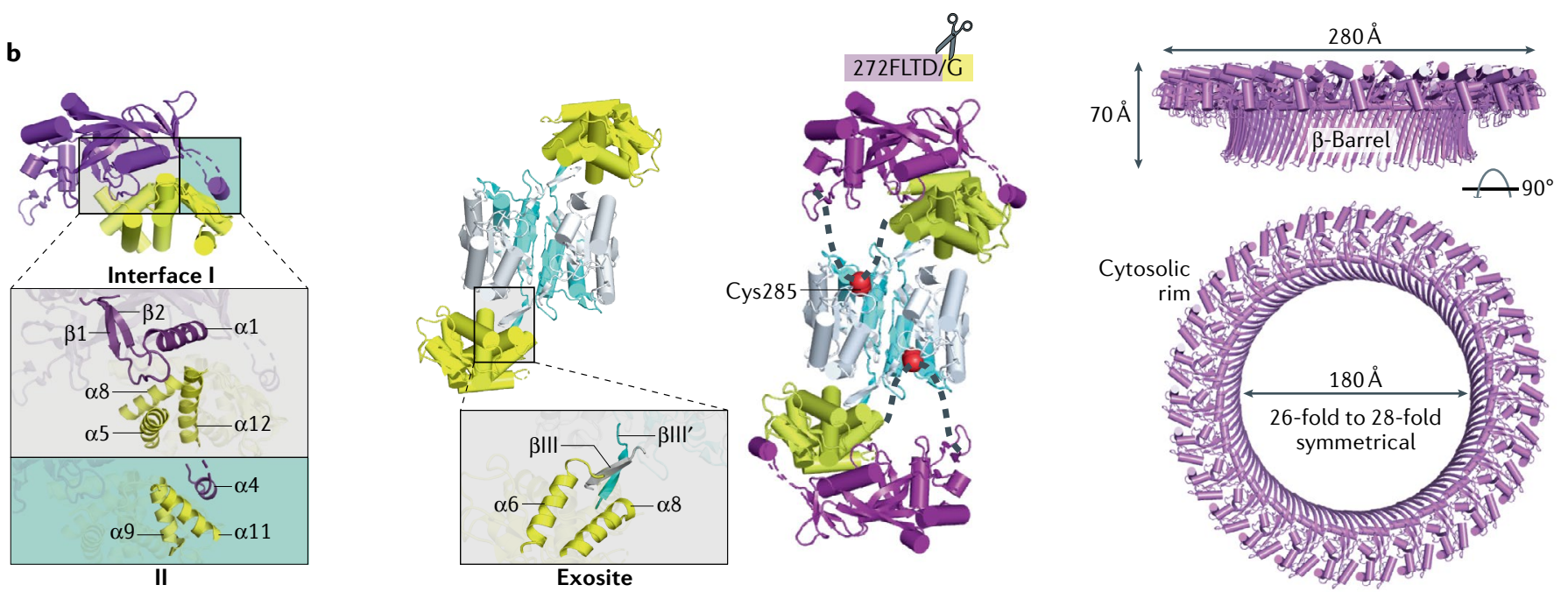

c

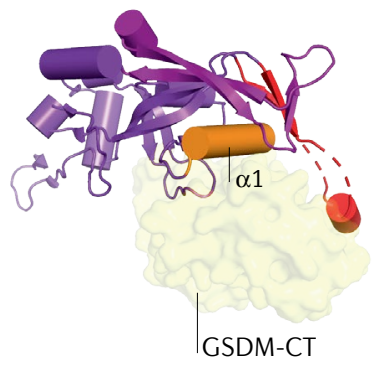

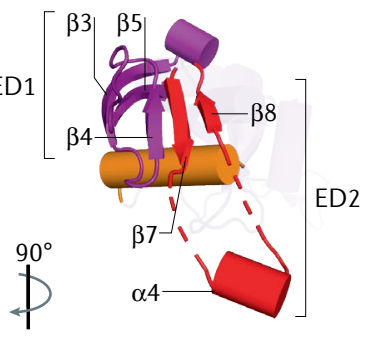

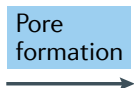

repair processes, which likely depends on how much GSDMD is in the cell and how quickly and efficiently it is cleaved. The latter will in turn depend on the intensity of inflammatory stimuli and degree of inflammasome and inflammatory caspase expression and activation, as well as the effectiveness of mechanisms for regulating and clearing inflammasomes, activated caspases and GSDMs. Given the importance of IL- $1 \beta$ secretion and pyroptosis in human inflammatory diseases, a better understanding of unconventional cytokine secretion and the regulation of pyroptosis is important.

Processing of IL-1 family cytokines removes an NT acidic region that converts pro-IL- $1 \beta$ from an acidic precursor into a more basic mature cytokine that binds to PIP2-rich acidic regions on the plasma membrane. Plasma

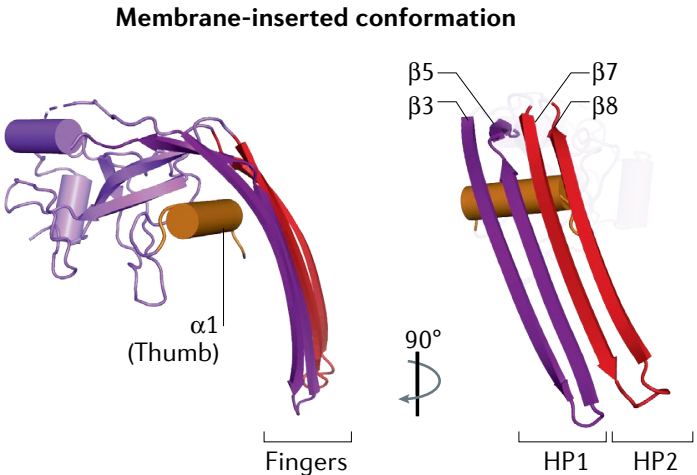

membrane association was proposed to be a necessary step for efficient release ${ }^{129}$. It is worth noting that $G s d m d^{-/-}$ macrophages can still release caspase 1-processed mature IL-1 $\beta$ but the GSDMD-independent release, which may also rely on PIP2-mediated plasma membrane binding, is much slower than GSDMD-dependent release ${ }^{129}$.

\section{Gasdermin-NTs and mitochondrial damage}

The activated NT domain has also been shown to bind to internal cellular membranes ${ }^{119,127,130,131}$. GSDMD-NT binds with apparently higher affinity to the mitochondrial and bacterial lipid, cardiolipin, than to plasma membrane lipids ${ }^{8,9}$. GSDMD-NT and GSDME-NT can translocate to mitochondria, permeabilize the outer membrane and disrupt mitochondrial function, leading 
4 Fig. 4 | Mechanisms of gasdermin auto-inhibition, processing and pore formation. a | Cartoon representation of gasdermin (GSDM) pore formation. Interaction between the $\mathrm{N}$-terminal and C-terminal fragments of a GSDM (GSDM-NT, in purple and GSDM-CT, in yellow) auto-inhibits protein activation. Active caspase 1, a dimer of the p10 (in pale blue)-p20 (in silver) complex, recognizes two copies of GSDM through the GSDM-CT. This exosite recognition mechanism places the long interdomain linker (in grey) between GSDM-NT and GSDM-CT near the active site of caspase 1 (red balls). Active caspase 4 and caspase 11 use a similar mechanism to process GSDMD. Linker cleavage liberates GSDM-NT for pore formation at the membrane. The fully formed GSDM pore features a large cytosolic rim in addition to a transmembrane $\beta$-barrel. $\mathbf{b} \mid$ Structural illustration of GSDM at each stage in pore formation depicted in part $\mathbf{a}$. In auto-inhibited GSDM (PDB: 5B5R for GSDMA3), GSDM-NT contacts GSDM-CT at two interfaces. Interface I is formed primarily by charged interactions of the $\alpha 1$ helix and the $\beta 1-\beta 2$ loop of GSDM-NT with the $\alpha 5-\alpha 8-\alpha 12$ helix cluster of GSDM-CT. Interface II is formed mainly by hydrophobic interactions between $\alpha 4$ of GSDM-NT and $\alpha 9$ and $\alpha 11$ of GSDM-CT. GSDMD recognition (PDB: $6 \mathrm{KN} 0$ and $6 \mathrm{VIE}$ ) by caspase 1 is mediated by a substrate-binding exosite, comprised of two anti-parallel $\beta$-strands - $\beta$ III of caspase 1-p20 and $\beta$ III' of caspase 1 -p10. The exosite binds to $\alpha 6$ and $\alpha 8$ of GSDMD-CT through primarily hydrophobic interactions. The GSDMD NT-CT linker is positioned near the active site Cys285 of caspase 1 for cleavage. The GSDM pore contains roughly 27 subunits according to the structure of the GSDMA3 pore (PDB: 6CB8). With each subunit contributing four transmembrane $\beta$-strands, the membrane-inserted $\beta$-barrel contains $108 \beta$-strands. The inner and outer diameters of the pore are approximately $180 \AA$ and $280 \AA$, respectively. A soluble rim, rich in $\alpha$-helices and loops, decorates the $\beta$-barrel on the cytosolic side. c | GSDM-NT conformations before (left) and after (right) pore formation. The positively charged lipid-binding helix $\alpha 1$ (in orange, like a thumb) is masked by GSDM-CT in the auto-inhibited conformation, and in the membrane-inserted conformation it interacts with lipids with negatively charged head groups found on the inner leaflet of the plasma membrane. Drastic conformational changes of GSDM-NT occur at extension domains 1 and 2 (ED1, in magenta and ED2, in red), which are located at the $\beta 3-\beta 4-\beta 5$ and $\beta 7-\alpha 4-\beta 8$ regions in auto-inhibited GSDM-NT, respectively. Through pore formation, ED1 and ED2 become the two transmembrane $\beta$-hairpins (HP1, in magenta and HP2, in red, like four fingers), respectively.

\section{NETosis}

A unique form of neutrophil cell death that is characterized by release of decondensed chromatin to form neutrophil extracellular traps (NETs) that trap microbes but can also capture and activate platelets to trigger clotting. to ROS generation, loss of transmembrane potential and cytochrome $c$ release, which also activates caspase 3 and enhances overall cell death ${ }^{119}$. As cardiolipin resides in the mitochondrial inner membrane, which is not accessible to the cytosol ${ }^{132}$, further study of mitochondrial targeting by GSDMs and its consequences is needed. NT fragments of GSDMA3, GSDMA and GSDME bind to the same phospholipids as GSDMD-NT ${ }^{9,17}$. Intriguingly, an in vitro lipid-binding assay showed that both full-length GSDMB and its NT domain bind to sulfatide and phosphoinositides, but not to cardiolipin ${ }^{133}$. The somewhat distinct phospholipid-binding properties of GSDMB suggest that it might bind to different membranes from the other GSDMs, but this has not been shown in cells.

\section{Role of gasdermin D in NETosis}

GSDMD plays a key role in NETosis, a regulated form of neutrophil cell death triggered by microbial infection, activation and danger signals, during which the nuclear envelope and plasma membrane are perforated to release large web-like structures, called neutrophil extracellular traps (NETs), composed of genomic DNA, histone and antimicrobial proteins ${ }^{43,131,134}$ (FIG. 3). In phorbol 12-myristate 13-acetate (PMA)-induced NET formation, serine proteases such as neutrophil elastase (ELANE) are released from granules, which cleave and activate GSDMD. GSDMD in turn permeabilizes primary azurophilic granules to further release ELANE in a feed-forward loop, allowing its nuclear translocation, histone processing, chromatin expansion and neutrophil lysis $^{43}$. Similarly, GSDMD is required for Gram-negative bacterial infection-triggered NETosis whereby LPS activates the noncanonical inflammasome, which activates GSDMD, which permeabilizes primary granules and the neutrophil plasma membrane to facilitate protease release, nuclear decondensation and NET extrusion ${ }^{131}$. Although chromatin is extruded through the nuclear envelope during NETosis, there is no evidence that activated GSDMD localizes to the nuclear envelope or is directly involved in its permeabilization.

In another study, the granule-associated protease cathepsin $\mathrm{G}$ (CatG) present in both macrophages and neutrophils cleaves GSDMD directly at Leu274, only two residues upstream of the caspase cleavage site at Asp276, to activate its pore-forming activity ${ }^{135}$ (FIG. 3). The catalytic activity of CatG on GSDMD appears to be stronger than that of other myeloid granule enzymes, including ELANE and proteinase 3, and even exceeds that of caspase 1 and caspase 11 , based on measured $k_{\text {cat }} / K_{\mathrm{M}}$ values. Sublethal LPS-induced systemic inflammation in mice depends on CatG and GSDMD and is suppressed by CatG-inhibiting serpins. Canonical NLRP3 inflammasome activation-dependent IL- $1 \beta$ release in bone marrow-derived macrophages (BMDMs) by nigericin or ATP also depends on CatG and GSDMD. Thus, GSDMD activation in both neutrophils and macrophages permeabilizes their granules to release CatG, which in turn further processes GSDMD in a feed-forward loop to enhance secretion of processed pro-inflammatory cytokines ${ }^{135}$.

\section{Gasdermins and disease}

Gasdermin D in infection. The affinity of GSDMD for bacterial cell membrane lipid cardiolipin allows it to bind, permeabilize and kill bacteria in the cytosol or phagosomes, as well as cell-free bacteria when it is released from pyroptotic cells ${ }^{8,9,89}$. Indeed, GSDMD was shown to mediate caspase 11-dependent defence against $S$. Typhimurium $\Delta$ sif $A^{41}$ and Brucella abortus ${ }^{42}$. Similarly, Gsdmd deficiency compromises AIM2-mediated host defence and makes mice susceptible to Francisella novicida infection ${ }^{40}$. GSDMD protects against Burkholderia pseudomallei using both canonical and noncanonical pathways ${ }^{89}$. For Legionella pneumophila infection, $G s d m d^{-/-}$mice showed only mildly increased susceptibility compared with wild-type mice and failed to recapitulate the greater susceptibility in $\mathrm{Nlrc}^{-/-}$mice $^{46}$. The NAIP5/NLRC4 inflammasome also activates caspase 8, which cleaves and activates caspase 7 , and GSDMD and caspase 7 together are responsible for restricting L. pneumophila infection ${ }^{46}$.

However, even though GSDMD-NT can permeabilize and kill bacteria, GSDMD is not always protective in infection and even exacerbates some bacterial infections by diverse mechanisms. In one study, Gsdmd $d^{-/-}$ mice were better able to survive pathogenic Escherichia coli infection because neutrophils, which are the main cellular defenders against bacteria ${ }^{43-45}$, survived longer without $G s d m d^{44}$. In another study, Mycobacterium tuberculosis infection in a human macrophage cell line induced plasma membrane damage and secondary activation of NLRP3 and GSDMD-dependent pyroptosis and inflammatory cytokine release, which increased 
bacterial cell-to-cell spread in vitro ${ }^{136}$. However, the overall effect of GSDMD and pyroptosis on mycobacterial infection would need to be studied in vivo.

In the case of parasites, Toxoplasma gondii has been reported in one study to induce AIM2, ASC and caspase 8-dependent, but GSDMD-independent, death in human macrophages ${ }^{137}$. However, another study suggests that $T$. gondii infection in human monocytes activates a Syk-CARD9-NF- $\kappa B$ signalling pathway and the NRLP3 inflammasome to release IL- $1 \beta$ in a GSDMD-independent manner ${ }^{138}$. IL- $1 \alpha$, but not IL-1 $\beta$, has been shown to be crucial for parasite control. IL-1 $\alpha$ release from microglia depends on GSDMD, and Gsdmd deficiency in mice reduces IL- $1 \alpha$ secretion and compromises immune protection in response to T. gondii infection in the brain ${ }^{139}$. GSDMD is activated in macrophages in vitro when another parasite, Entamoeba histolytica, binds and activates the noncanonical caspase 4 inflammasome by sensing an unknown ligand ${ }^{140}$. In vitro, E. histolytica causes caspase 4 and GSDMD-dependent macrophage hyperactivation with IL- $1 \beta$ secretion but without pyroptosis. Determining whether the net physiological impact of GSDMD is to improve immune control or exacerbate tissue damage in E. histolytica infection will require in vivo experiments.

The role of inflammasome activation and GSDMD in viral infection is not well studied. Viruses activate Toll-like receptors that sense viral nucleic acids on the cell surface or in endosomes to prime expression of innate immune genes. Cytoplasmic DNA and RNA sensors can trigger an interferon response (that is, cGAS, RIGI and MDA5) and/or an inflammatory response (IFI16, AIM2, NLRP6 and NLRP9) to viruses. Many viral proteins inhibit the antiviral interferon response, but so far viral mechanisms to inhibit inflammasome activation and pyroptosis have not been identified. In one study, the NLRP9 inflammasome, which recognizes stretches of viral dsRNA, protected intestinal epithelial cells from rotavirus infection in a GSDMD-dependent manner ${ }^{141}$. By contrast, inflammasome and GSDMD activation might contribute to viral pathogenesis for viruses whose disease course has a strong inflammatory component (such as HIV, influenza A or SARS coronaviruses). In rhinovirus and norovirus infections, NLRP3- and GSDMD-dependent pyroptosis increases pathological inflammation, contributing to viral pathogenesis ${ }^{90,142}$.

Gasdermin D in autoinflammatory genetic diseases. Autoactivation of inflammasome pathways causes or contributes to multiple sterile inflammatory diseases. Familial Mediterranean fever (FMF), the most prevalent monogenic autoinflammatory disease worldwide, is caused by missense mutations in MEFV. MEFV encodes the pyrin inflammasome, which senses bacterial toxin inactivation of RhoA GTPases ${ }^{143}$. MEFV mutation reduces the threshold for activating the pyrin inflammasome, causing recurrent bouts of fever and pain. GSDMD is crucial for FMF autoinflammatory pathology. In a mouse FMF model that expresses a hypersensitive pyrin, Gsdmd deficiency abolishes auto-inflammatory symptoms ${ }^{144}$. Similarly, autoactive NLRP3 mutants result in autoinflammatory disorders, including NOMID, which features severe systemic inflammation that is not adequately treated with current cytokine inhibitor drugs. Ablation of Gsdmd in NOMID mice prevents disease symptoms ${ }^{26}$ (FIG. 5).

Gasdermin D in complex diseases exacerbated by inflammation. GSDMD is probably the most important GSDM in mediating the inflammation that is associated with many diseases. It is the convergent target of canonical and noncanonical inflammasome signalling in immune sentinel cells, and excessive activation of GSDMD-mediated pyroptosis and IL-1 family cytokine secretion can lead to inflammatory diseases and pathological consequences (FICS 4,5). An unrestrained inflammatory response to infection is responsible for cytokine release syndrome (CRS), sepsis, disseminated intravascular coagulation (DIC) and acute organ damage, including acute respiratory distress syndrome (ARDS) and acute kidney injury (AKI). Release of IL-1 family cytokines is crucially dependent on GSDMD activation ${ }^{21,122}$. Binding of these cytokines to the IL-1 receptor triggers the activation and release of other pro-inflammatory cytokines, including IL-6 and TNF ${ }^{145}$. GSDMD-mediated pyroptosis in macrophages also triggers release of tissue factor, which activates coagulation, leading to DIC in sepsis ${ }^{146,147}$. Similarly, GSDMD-dependent neutrophil NETs capture platelets and serve as a nidus for thrombus formation, which can also contribute to $\mathrm{DIC}^{148}$. The noncanonical inflammasome plays a key role in sepsis in mouse models ${ }^{14,104,149}$. In LPS and caecal ligation and puncture (CLP)-induced sepsis, Gsdmd deficiency protects mice from lung, intestine, liver and kidney injury and DIC ${ }^{14,150}$. CRS and ARDS, triggered in patients with clinical deterioration during pathogenic influenza and SARS respiratory infections, could also be mediated or exacerbated by pyroptosis in tissue-resident macrophages, but this has not been shown.

GSDMD has been clearly linked to noninfectious diseases that are not caused by a single genetic mutation, in which inflammation plays an important pathogenic role. The NLRP3 inflammasome is implicated in EAE in mice. Gsdmd deficiency suppresses EAE neuroinflammation, and GSDMD inhibitors disulfiram (DSF) and dimethyl fumarate (DMF) attenuate $\mathrm{EAE}^{25,48}$. In EAE, GSDMD-mediated pyroptosis is activated in peripheral myeloid cells as well as in microglia and oligodendrocytes in the $\mathrm{CNS}^{25,151}$, but which cell types are most crucial for neuroinflammation is unclear. In a mouse bone marrow transplantation model, a recent study indicated that radiation causes severe damage to bones and spleen through NLRP3- and AIM2-mediated GSDMD-dependent pyroptosis, which is prevented only when GSDMD is deficient in both donor and host cells ${ }^{152}$. Activation of the noncanonical inflammasome pathway in mice worsens graft-versus-host disease, and Gsdmd deficiency reduces intestinal inflammation, tissue damage, donor $\mathrm{T}$ cell expansion and mortality in allogeneic haematopoietic stem cell transplantation ${ }^{153}$. Genetic deficiency or knockdown studies have also implicated GSDMD in the pathogenesis of alcoholic hepatitis, nonalcoholic steatohepatitis, noninfectious liver injury and ischaemia-reperfusion injury in humans and mice $^{154-158}$. Both caspase 8 and GSDMD have also been 
implicated in mixed-lineage kinase domain-like protein (MLKL)-independent ileitis in mice with intestinal epithelial FADD deficiency ${ }^{108}$. The serum of children with acute vasculitis in Kawasaki disease contains elevated levels of GSDMD and IL- $1 \beta$ and induces endothelial cell pyroptosis in vitro, suggesting that GSDMD-mediated pyroptosis plays an important role in endothelial damage in Kawasaki disease ${ }^{159}$. GSDMD-mediated pyroptosis has also been implicated in diabetic kidney disease ${ }^{160}$, diabetic cardiomyopathy ${ }^{161}$, contrast-induced kidney injury ${ }^{162}$, early brain injury after subarachnoid haemorrhage ${ }^{163}$ and traumatic brain injury ${ }^{164}$. Although activation of canonical and noncanonical inflammasomes has been implicated in the inflammation and pathogenesis of many noninfectious human diseases, the role of GSDMD in all these conditions has not yet been verified by studies in Gsdmd-deficient mice.

Gasdermins in cancer. The role of GSDMs in cancer is complicated (reviewed elsewhere ${ }^{47}$ ). The 2000 paper $^{5}$ that named GSDMA based on its restricted expression in the gastrointestinal tract and skin hinted at a possible role of GSDMs in cancer by noting that GSDMA expression is suppressed in human gastric cancers and that it might be a tumour suppressor gene ${ }^{5}$. In fact, GSDMA, GSDMC and GSDMD all reportedly suppress gastric cancer cell proliferation ${ }^{70,165}$. However, reduced GSDMD expression attenuates tumour proliferation and predicts a favourable prognosis in non-small-cell lung cancer ${ }^{166}$. $G S D M B$ is amplified and highly expressed in cervical,

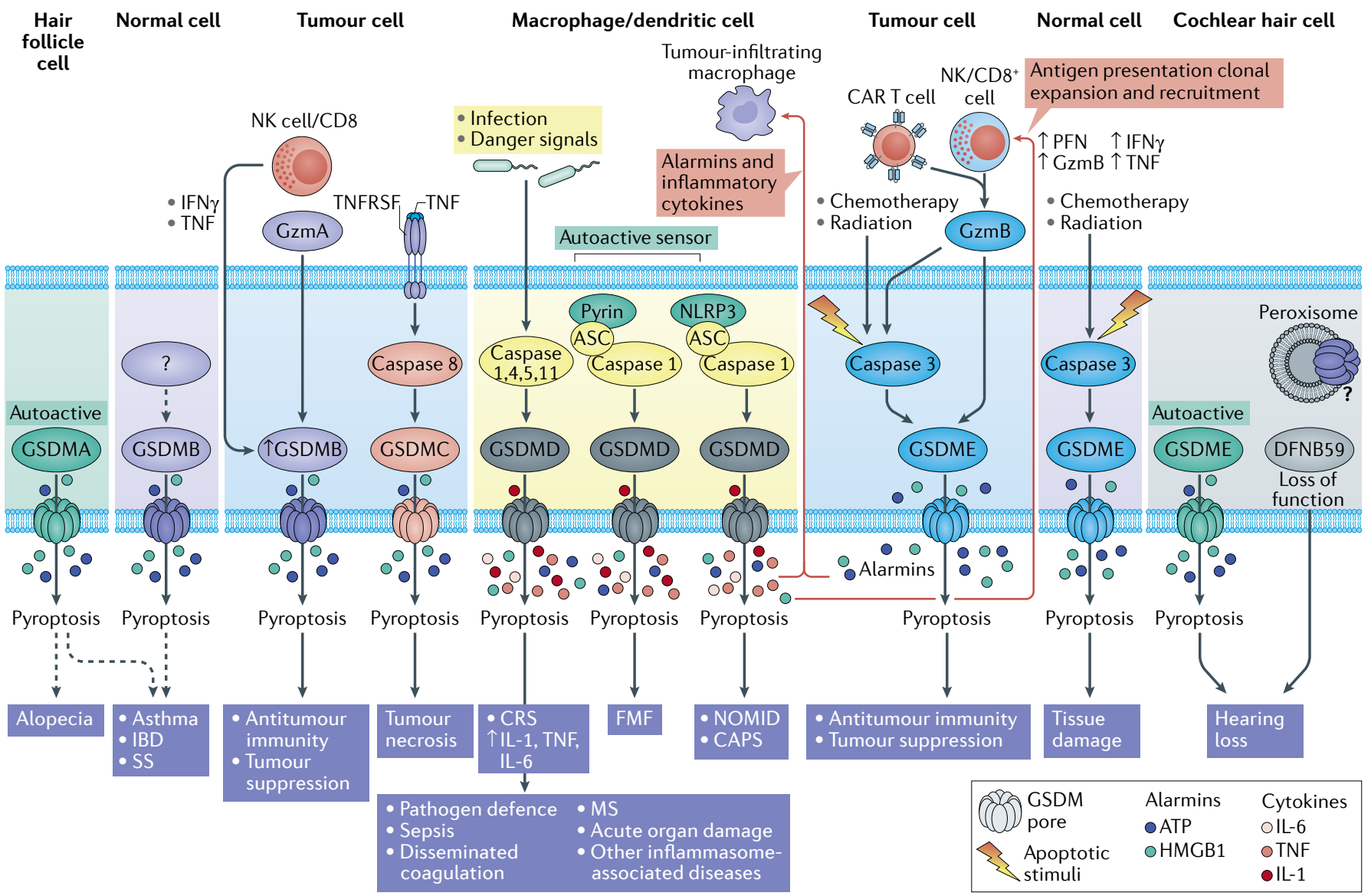

Fig. 5 | Role and function of gasdermins in human disease. Autoactive gasdermin A (GSDMA) triggers pyroptosis in hair follicle cells and causes alopecia in mice but so far no human link to alopecia has been reported. GSDMB is implicated in autoimmune diseases such as childhood asthma and inflammatory bowel disease (IBD), but the underlying mechanism remains unclear. Granzyme A (GzmA) in killer lymphocytes activates GSDMB in target cells to cause pyroptosis. TNF can activate caspase 8 to trigger GSDMC-mediated pyroptosis in tumours. Upon sensing infection or danger, assembly of inflammasomes activates inflammatory caspases to cleave GSDMD and trigger pyroptosis, initiating immune responses. However, excessive pyroptosis leads to lethal sepsis, cytokine release syndrome (CRS) and severe inflammation and tissue damage. Autoactive pyrin or NLRP3 constitutively activate GSDMD and cause pyroptosis leading to familial Mediterranean fever (FMF) and neonatal-onset multisystem inflammatory disease (NOMID), respectively. Natural killer (NK) and CD8 ${ }^{+}$killer lymphocytes and chimeric antigen receptor (CAR) T cells activate pyroptosis in GSDME-expressing tumours when GzmB cleaves and activates GSDME and caspase 3 . Alarmins released from pyroptotic tumour cells can also activate GSDMD-mediated pyroptosis in tumour-infiltrating macrophages and dendritic cells to enhance antigen presentation and functional activation of tumour-infiltrating lymphocytes (TILs). Secondary activation of macrophages can also mediate CRS during CAR T cell therapy. Chemotherapy drugs and radiotherapy activate caspase 3 in GSDMEexpressing cells to convert apoptosis into pyroptosis, increasing their effectiveness at suppressing tumour growth by activating antitumour immunity; at the same time normal tissue cell damage and cytotoxicity is increased by activating GSDME-dependent pyroptosis especially in gut epithelial cells and haematopoietic precursor cells. Autoactive GSDME induces cochlear hair cell pyroptosis, leading to nonsyndromic hearing impairment. Loss-of-function mutation of DFNB59 also causes recessive nonsyndromic hearing loss. CAPS, cryopyrin-associated periodic syndromes; MS, multiple sclerosis; SS, systemic sclerosis. 
hepatic, colon and HER2 ${ }^{+}$breast cancer and may function as an oncogene in those cancers ${ }^{71,79}$. GSDMB expression in HER2 ${ }^{+}$breast cancer correlates with increased metastasis and poor prognosis ${ }^{78,80}$. Overexpression of GSMDB in HER2 ${ }^{+}$MCF7 cells promotes cell mobility and invasion, probably by activating Racl and $C d c 42$ $\left(\mathrm{REF}^{78}\right)$. Moreover, introducing anti-GSDMB into HER2 ${ }^{+}$ tumour cells using a hyaluronan-coated nanoparticle reduces xenograft growth and metastasis in vivo ${ }^{167}$. By contrast, GSDMB expression is suppressed in primary oesophageal and gastric tumours ${ }^{19}$, and its expression correlates with better outcome in patients with bladder carcinoma or melanoma. GSDMC is upregulated in melanoma, colorectal cancer and lung adenocarcinoma, promotes tumour growth and metastasis, and might function as an oncogene in those cancers ${ }^{82,85,168}$. These studies suggest that the roles of GSDMs in cancer are context, GSDM and cancer type specific, reflecting the complex role of inflammation in tumorigenesis, cancer proliferation and antitumour immunity ${ }^{169}$. Because of this complexity, these studies need to be interpreted with caution. In vitro experiments and studies in immunodeficient mice do not take into account the interaction between the tumour and the immune system, where GSDMs may play their most important in vivo role.

GSDME, which is activated by caspase 3 during apoptosis, is more strongly implicated in tumour suppression. GSDME is epigenetically suppressed by promoter DNA methylation in gastric, colorectal and breast cancers and most human cancer cell lines; and most tumour-associated GSDME mutations studied compromise its ability to induce pyroptosis ${ }^{17,18,56-60}$ (TABLE 2). Moreover, GSDME expression, induced by 5 -aza-dC in vitro, suppresses colony formation and tumour cell proliferation in gastric cancer, melanoma and colorectal cancer, and reduces invasivity of breast cancer ${ }^{56,57,60,119}$. Worse 5-year survival and an increase in lymph node metastases are associated with reduced GSDME in breast cancer ${ }^{57}$. Moreover, lack of GSDME promotes melanoma cell line resistance to etoposide, which is rescued by GSDME overexpression ${ }^{170}$. In addition, treatment of lung cancer cells with KRAS, EGFR or ALK inhibitors leads to caspase 3-mediated GSDME activation, which increases the antitumour effectiveness of these drugs ${ }^{171}$.

The tumour-suppressive effect of GSDME is dependent on immune surveillance in vivo by converting $\mathrm{NK}$ and $\mathrm{CD}^{+} \mathrm{T}$ killer cell-mediated noninflammatory apoptosis into inflammatory pyroptosis in the tumour, which augments antitumour immunity ${ }^{18}$ (FIGS 3,5). This effect is abrogated in tumours that express noncleavable GSDME and in mice that lack all lymphocytes, only killer lymphocytes or perforin that is needed for cytotoxic granule-mediated killing ${ }^{18}$. Gsdme expression in mouse tumours suppresses tumour growth and enhances the number and functions of tumour-infiltrating NK and $\mathrm{CD}^{+} \mathrm{T}$ lymphocytes ${ }^{18}$. When killer cells recognize a tumour target, they deliver the cytotoxic protease GzmB into the tumour, which induces GSDME-mediated pyroptosis, both directly because GzmB cleaves and activates GSDME and indirectly because GzmB also cleaves and activates caspase $3\left(\mathrm{REF}^{18}\right)$. Similar findings were reported using chimeric antigen receptor (CAR) T cells, which induce pyroptosis in GSDME-expressing target cells, but how much GSDME pyroptosis contributes to the effectiveness of CAR T cell therapy has not been evaluated ${ }^{172}$. Caspase 3 activation by GzmB generates a positive feedback mechanism as caspase 3 also cleaves and activates GSDME. As GSDME can be cleaved and activated by both caspase 3 and GzmB, GSDME may be a more potent tumour suppressor than the other GSDMs. Caspase 3 activation is common in cancers, triggered by an inadequate supply of oxygen or nutrients, especially in poorly vascularized regions, by treatment with chemotherapy or radiation and by killer cell attack. Because GSDME is suppressed by promoter hypermethylation in many cancers $^{53-57}$, epigenetic modifying drugs could promote antitumour immunity. Indeed, combining 5 -aza-dC with chemotherapy in one study enhanced the immunogenic effect of chemotherapy ${ }^{173}$. However, it is unclear how much pyroptosis contributes to the 5-aza-dC-induced antitumour effect as 5-aza-dC should derepress many genes, including tumour suppressor genes.

The potential therapeutic role of inducing pyroptosis in tumours has been demonstrated in a number of examples. Like GzmB-induced GSDME activation, the killer lymphocyte cytotoxic protease GzmA cleaves GSDMB and induces pyroptosis in tumour cells targeted for immune elimination ${ }^{19}$ (FICS 3,5). When combined with anti-PD1, ectopic expression of human GSDMB in murine colorectal cancer CT26 and melanoma B16 promotes tumour clearance in mice. The effectiveness of BRAF and MEK inhibitors against GSDME-expressing $B R A F^{\mathrm{V} 600 \mathrm{E} / \mathrm{K}}$ mutant melanoma was shown to depend on the induction of GSDME-mediated pyroptosis to activate $\mathrm{CD}^{+} \mathrm{T}$ cell-dependent antitumour immunity ${ }^{174}$. In another study, the serine dipeptidase DPP8/9 inhibitor Val-boroPro, which activates the CARD8 inflammasome in human myeloid cells, induced GSDMD-mediated pyroptosis in most human acute myeloid leukaemia (AML) cell lines and primary patient samples, but not cell lines from other lineages ${ }^{175}$.

These data suggest that GSDM-mediated pyroptosis plays a crucial role in antitumour immunity for some tumours, and harnessing this process could be an effective way to treat cancer. However, activating GSDMs in GSDM-expressing normal tissues and immune cells can also cause severe tissue damage, such as in chemotherapyinduced caspase 3-mediated GSDME activation in normal tissues ${ }^{17}$ (FIGS 3,5). Although a DPP8/9 inhibitor induces pyroptosis in AML cells, it may also activate GSDMD-mediated pyroptosis in B cells and other $\mathrm{CD} 4^{+}$cells ${ }^{175}$ and cause treatment-associated toxicity. Activation of GSDME-mediated pyroptosis in normal mouse tissues also causes extensive tissue damage after chemotherapy ${ }^{17}$. During CAR T cell anticancer therapy of B cell leukaemia, GzmB activates both GSDME and caspase 3, causing pyroptosis. Moreover, culture supernatants from B-ALL human cell lines or primary leukaemias cocultured with CAR T cells activate caspase 1-GSDMD-mediated pyroptosis in macrophages. Activation of pyroptosis by CAR T cells is responsible for CRS, one of the serious side effects of this therapy ${ }^{172}$ (FICS 3,5). Thus, selective activation of GSDMs in tumours 

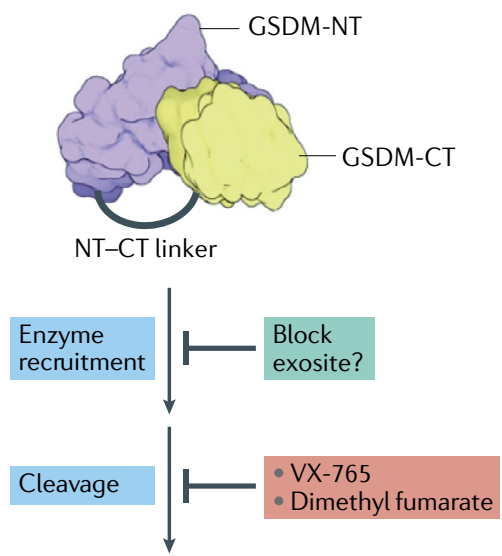

NT+CT non-covalent complex
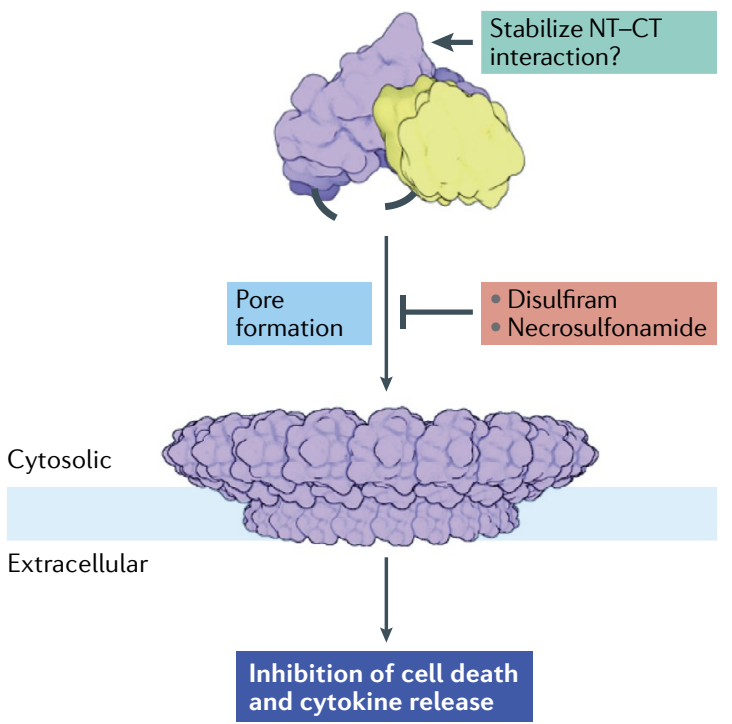
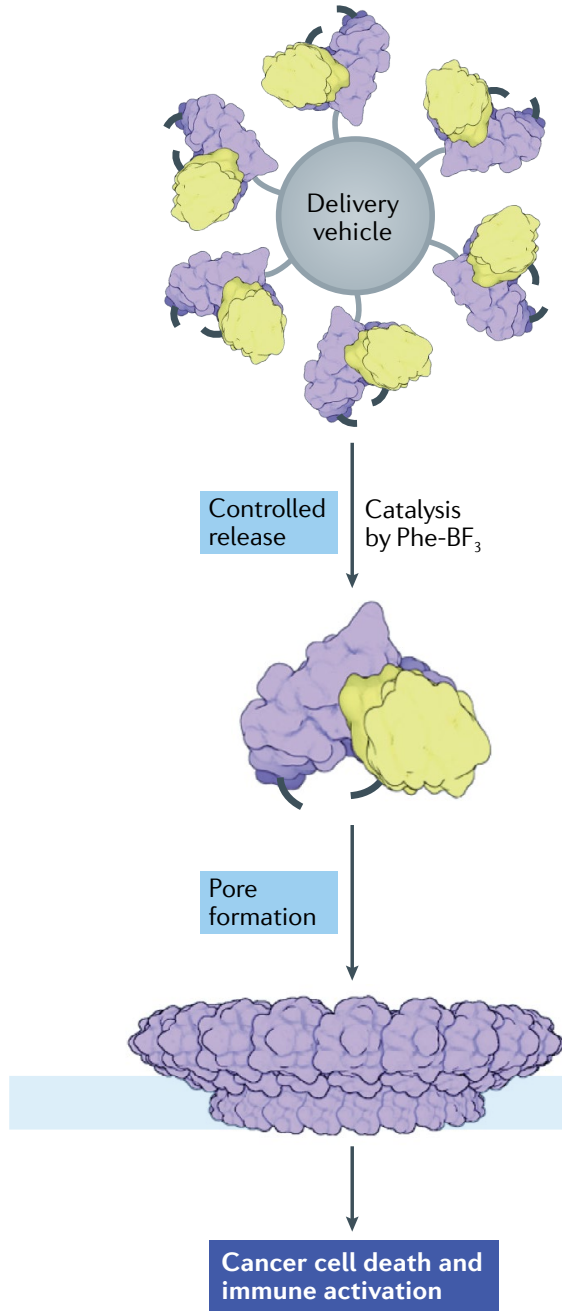

Fig. 6 | Strategies to treat gasdermin-associated diseases. a | Steps in gasdermin (GSDM) activation that can be targeted, and the mechanisms used by existing GSDMD inhibitors ${ }^{45,48}$. Other yet unused potential mechanisms are shown with a question mark. $\mathbf{b}$ |Cellular delivery of GSDM for cancer cell killing and activation of antitumour immunity ${ }^{180}$. See text for details. CT, C-terminal; NT, N-terminal.

rather than normal cells could be crucial for developing pyroptosis-inducing cancer therapy.

\section{Therapeutically targeting gasdermins}

The key roles of GSDMs in infection, inflammation and tumour suppression have prompted investigation of GSDM-targeting therapeutics (FIG. 6). Inflammasome pathways have already been extensively targeted indirectly for disease modulation by inhibiting IL-1 $\beta$, the signature cytokine released in a GSDMD activation-dependent manner, or its receptor, using the FDA-approved drugs anakinra, a recombinant natural human IL-1 receptor antagonist, and canakinumab, an anti-IL- $1 \beta$ monoclonal antibody ${ }^{176}$. These drugs are used to treat severe manifestations of autoinflammatory diseases, including FMF, NOMID, severe systemic autoimmune diseases (rheumatoid arthritis, systemic juvenile idiopathic arthritis, adult-onset Still's disease) and bronchiolitis obliterans that do not respond to other treatments. Small-molecule inhibitors of caspase 1, such as VX-765, have also been developed ${ }^{151}$, although they have not proved useful for treating human disease. Inhibitors of inflammasome sensors are under clinical development ${ }^{177}$. However, GSDMD is an especially attractive drug target because it is the bottleneck for all inflammasome effects, acting upstream of IL-1 and alarmin secretion and downstream of all inflammasome sensors (FIGS 2,3,5). Inhibition of GSDMD and possibly other GSDMs may therefore be the most efficacious strategy for treating diseases that are primarily inflammatory and/or autoimmune and may also be useful for treating diseases exacerbated by pyroptosis, such as cardiovascular disease and CRS in severe COVID-19 infection. On the other hand, induction of GSDM-mediated cell death may be useful for amplifying immune responses, such as in vaccination or cancer.

Small-molecule gasdermin inhibitors. A few studies have identified small-molecule GSDMD inhibitors (TABLE 3). DSF, an FDA-approved drug for treating chronic alcoholism (Antabuse), was identified in a 
Table 3 | Gasdermin D inhibitors

\begin{tabular}{|c|c|c|c|c|c|c|}
\hline GSDMD inhibitor & Description & In vitro $\mathrm{IC}_{50}$ & Cellular IC & $\begin{array}{l}\text { Mechanism of } \\
\text { action }\end{array}$ & In vivo effect & Refs \\
\hline Disulfiram & $\begin{array}{l}\text { Commonly } \\
\text { known as } \\
\text { Antabuse, an } \\
\text { FDA-approved } \\
\text { alcoholism } \\
\text { drug }\end{array}$ & $\begin{array}{l}\text { 300 nM } \\
\text { alone in } \\
\text { GSDMD- } \\
\text { induced } \\
\text { liposome } \\
\text { leakage; } \\
\sim 200 \mathrm{nM} \text { in } \\
\text { combination } \\
\text { with Cu(II) }\end{array}$ & $\begin{array}{l}\sim 7.7 \mu \mathrm{M} \text { in } \\
\text { canonical } \\
\text { inflammasome- } \\
\text { dependent } \\
\text { pyroptosis in } \\
\text { THP-1; } 10.3 \mu \mathrm{M} \\
\text { in noncanonical } \\
\text { inflammasome- } \\
\text { dependent } \\
\text { pyroptosis in } \\
\text { murine iBMDMs; } \\
\sim 400 \text { nM in } \\
\text { combination with } \\
\text { Cu(II) in THP-1 }\end{array}$ & $\begin{array}{l}\text { Binds GSDMD } \\
\left(K_{d}=\sim 12.8 \mu M\right), \\
\text { covalently } \\
\text { modifies Cys191 } \\
\text { in GSDMD, } \\
\text { and inhibits } \\
\text { GSDMD-NT } \\
\text { oligomerization/ } \\
\text { insertion }\end{array}$ & $\begin{array}{l}\text { Increased } \\
\text { survival and } \\
\text { suppression of } \\
\text { inflammatory } \\
\text { responses in } \\
\text { murine models } \\
\text { of sepsis and } \\
\text { EAE }\end{array}$ & 25,48 \\
\hline Necrosulfonamide & $\begin{array}{l}\text { An inhibitor } \\
\text { of MLKL in } \\
\text { necroptosis }\end{array}$ & $\begin{array}{l}\sim 9.5 \mu \mathrm{M} \text { in } \\
\text { GSDMD- } \\
\text { induced } \\
\text { liposome } \\
\text { leakage }\end{array}$ & $\begin{array}{l}\sim 10-15 \mu \mathrm{M} \\
\text { in canonical } \\
\text { inflammasome- } \\
\text { dependent } \\
\text { pyroptosis in } \\
\text { iBMDMs }\end{array}$ & $\begin{array}{l}\text { Binds GSDMD } \\
\left(K_{d}=\sim 32 \mu M\right), \\
\text { potentially } \\
\text { modifies Cys191 } \\
\text { in GSDMD, } \\
\text { and inhibits } \\
\text { GSDMD-NT } \\
\text { oligomerization/ } \\
\text { insertion }\end{array}$ & $\begin{array}{l}\text { Increased } \\
\text { survival and } \\
\text { suppression of } \\
\text { inflammatory } \\
\text { responses in a } \\
\text { murine model } \\
\text { of sepsis }\end{array}$ & 45,48 \\
\hline Dimethyl fumarate & $\begin{array}{l}\text { An FDA- } \\
\text { approved } \\
\text { drug } \\
\text { (Tecfidera) for } \\
\text { the treatment } \\
\text { of MS }\end{array}$ & NA & NA & $\begin{array}{l}\text { Succinates } \\
\text { GSDMD at } \\
\text { Cys191 and } \\
\text { other reactive } \\
\text { cysteines, } \\
\text { prevents } \\
\text { interaction } \\
\text { between GSDMD } \\
\text { and caspases, and } \\
\text { inhibits } \\
\text { GSDMD-NT } \\
\text { oligomerization/ } \\
\text { insertion }\end{array}$ & $\begin{array}{l}\text { Increased } \\
\text { survival and } \\
\text { suppression of } \\
\text { inflammatory } \\
\text { responses in } \\
\text { murine models } \\
\text { of sepsis, EAE } \\
\text { and FMF; } \\
\text { decreased } \\
\text { levels of } \\
\text { GSDMD-NT } \\
\text { and IL-1 } \beta \text { in } \\
\text { patients with } \\
\text { MS treated with } \\
\text { Tecfidera }\end{array}$ & 48,49 \\
\hline Punicalagin & $\begin{array}{l}\text { A complex } \\
\text { polyphenol } \\
\text { from } \\
\text { pomegranate } \\
\text { extract }\end{array}$ & NA & $\begin{array}{l}\sim 3.9,3.7 \text { and } \\
7.5 \mu \mathrm{M} \text { in IL-1 } \beta \\
\text { secretion, LDH } \\
\text { release and } \\
\text { membrane } \\
\text { permeabilization, } \\
\text { respectively, } \\
\text { in ATP-treated } \\
\text { murine BMDMs }\end{array}$ & $\begin{array}{l}\text { Potentially } \\
\text { interferes with } \\
\text { membrane } \\
\text { fluidity and } \\
\text { inhibits } \\
\text { GSDMD-NT } \\
\text { insertion }\end{array}$ & NA & 179 \\
\hline
\end{tabular}

The half-maximal inhibitory concentration $\left(\mathrm{IC}_{5}\right)$ and equilibrium dissociation constant $\left(K_{\mathrm{d}}\right)$ values were taken from the referenced studies. EAE, experimental autoimmune encephalomyelitis; FMF, Familial Mediterranean fever; GSDM, gasdermin; iBMDMs, immortalized murine bone marrow-derived macrophages; $\mathrm{LDH}$, lactate dehydrogenase; MLKL, mixed-lineage kinase domain-like protein; NA, not available; NT, N-terminal; MS, multiple sclerosis; PMA, phorbol myristate acetate; THP-1, a human monocytic cell line. 
high-throughput liposome leakage-based screen as an inhibitor of GSDMD pore formation on liposomes with a half-maximal inhibitory concentration $\left(\mathrm{IC}_{50}\right)$ in the nanomolar range ${ }^{48}$. In human THP-1 cells and mouse immortalized BMDMs (iBMDMs), stimulated by the canonical NLRP3 and AIM2 inflammasomes and the noncanonical inflammasome, respectively, DSF blocked pyroptosis and cytokine release without affecting inflammasome speck formation, caspase activation or GSDMD cleavage, suggesting that it was acting by blocking pore formation. In a mouse model of LPS-induced septic shock, DSF protected wild-type and caspase 1-knockout mice from death and greatly reduced serum inflammatory cytokine levels. The potency of DSF was enhanced when it was combined with copper gluconate, which reportedly stabilizes diethyldithiocarbamate (DTC), the cellular metabolite of DSF ${ }^{178}$. The efficacy of DSF in protecting against GSDMD-mediated inflammatory disorders was also demonstrated in $\mathrm{EAE}^{25}$. Necrosulfonamide (NSA), a drug previously known to inhibit MLKL in necroptotic cell death, was also shown to inhibit GSDMD pore formation, with low micromolar cellular $\mathrm{IC}_{50}$ in blocking cytokine release and pyroptosis downstream of NLRP3 and NLRC4 inflammasomes ${ }^{45}$. Like DSF, NSA extended survival of wild-type mice in a model of LPS-induced sepsis.

DMF, FDA-approved Tecfidera for the treatment of MS, was reported to prevent GSDMD from interacting with caspases, limit GSDMD processing and oligomerization, and block pyroptosis; endogenous fumarate similarly inhibited GSDMD ${ }^{49}$. In murine models of sepsis, EAE and FMF, DMF extended survival and alleviated inflammation. Patients with MS treated with DMF exhibited decreased plasma GSDMD-NT and IL- $1 \beta$ compared with untreated controls, consistent with a model in which GSDMD exacerbates MS, as was shown in mouse EAE. These strong inhibitory effects of DMF contrast with the lack of inhibition of GSDMD-induced liposome leakage by DMF reported in an earlier study ${ }^{48}$. These two studies suggest that DMF may inhibit GSDMD cleavage, while DSF and NSA work downstream in the pathway to inhibit pore formation. Further characterization of DMF is necessary to evaluate its potency as a GSDMD inhibitor.

DSF, NSA and DMF are all Cys-reactive drugs that inhibit GSDMD by modifying Cys residues (TABLE 3). DSF and NSA covalently modify Cys191, but not other cysteines, in GSDMD to inhibit pore formation ${ }^{45,48}$, while DMF and endogenous fumarate modify multiple Cys residues, including Cys191, leading to GSDMD succination $^{49}$. Consistently, GSDMD with mutated Cys191 and other GSDMs that lack this reactive cysteine are not inhibited by DSF or NSA ${ }^{45,48}$. GSDMD Cys 191 is an exposed cysteine on the $\alpha 4$ helix that plays an important role in membrane penetration (FIG. 4b). Modification of Cys191 may interfere with membrane insertion and therefore abrogate formation of transmembrane $\beta$-hairpins (FIGS 4c,6a). Although the exposed position of Cys191 may facilitate its modification, a downside of Cys-reactive compounds is that they are less selective and likely also target molecules other than GSDMD. Therefore, the mechanisms of action of DSF, NSA and DMF in vivo should be interpreted with caution.
Two other molecules, LDC7559, a pyrazolooxazepine-based compound, and punicalagin, an antioxidant polyphenol from pomegranates, have been reported to inhibit PMA-induced NETosis and NLRP3/ AIM2 inflammasome-dependent pyroptosis, respectively, with activity in the low micromolar range ${ }^{43,179}$. In pulldown experiments using cell lysates, LDC7559 specifically enriched GSDMD detection by mass spectrometry. However, the interaction between LDC7559 and GSDMD was not directly verified, and LDC7559 does not inhibit GSDMD pore formation on liposomes ${ }^{48}$, hence leaving its mechanism of action unexplained. Likewise, considering that punicalagin also protects macrophages from detergents and may indirectly affect GSDMD pore formation by rigidifying the plasma membrane, further studies are needed to elucidate whether punicalagin directly and specifically binds to GSDMD.

On the basis of a structural understanding of GSDM auto-inhibition, molecules that stabilize the GSDM NTCT interface may inhibit the full-length protein by locking it in its inactive conformation (FIG. 6a). The recent identification of an exosite for inflammatory caspase recognition of GSDMD suggests that another possible way of inhibiting GSDMD would be to identify a compound that blocks the exosite (FIG. 6a). It is likely that other post-translational modifications and mechanisms to regulate pore formation will be uncovered that might be attractive drug targets.

Manipulating gasdermin expression in cells. In an effort to induce GSDM-mediated cancer cell pyroptosis to prime adaptive immunity, intratumoural delivery of nanoparticle-conjugated, pre-cleaved GSDMA3 $(\mathrm{NT}+\mathrm{CT})$ into cells was combined with the cancerimaging compound phenylalanine trifluoroborate $\left(\mathrm{Phe}-\mathrm{BF}_{3}\right)$, which catalyses the release of GSDMA3 from nanoparticles, causing selective tumour cell pyroptosis ${ }^{180}$ (FIG. 6b). Pyroptosis of $<15 \%$ of tumour cells was sufficient to suppress the entire tumour graft in mice, because the treatment activated the adaptive immune response to the tumour. Immunodeficiency or T cell depletion abrogated the tumour-suppressive response. Further, a partially effective intravenous treatment with the nanoparticles synergized with checkpoint blockade and sensitized the tumours to anti-PD1, suggesting that pyroptosis could be exploited to trigger or enhance antitumour immunity. This study provides a proof of concept for targeting GSDMs in cancer; however, care needs to be taken to avoid excessive pyroptosis, which could be toxic to normal cells or cause cytokine release with systemic consequences. Delivery of GSDM-NT or identification of GSDM agonists could also in principle be used as vaccine adjuvants or to enhance the immune response to infection.

\section{Considerations for therapeutic GSDMD inhibition} The wide variety of diseases in which GSDMD likely mediates inflammation and its crucial role as a final bottleneck for pyroptosis suggest that GSDMD inhibition could be an extremely effective therapeutic strategy. However, interfering with the antimicrobial roles of GSDMD could lead to increased susceptibility to 
infection. In addition, innate immune responses are complex, and each potential therapeutic indication will need to be examined carefully in preclinical models. In a study using the monosodium urate (MSU)-induced gout model, although GSDMD was activated through the NLRP3 inflammasome, it was not needed for cell death or IL- $1 \beta$ release, and the GSDMD inhibitor NSA did not ameliorate disease ${ }^{181}$. Suppressing pyroptosis can activate other innate immune responses. For instance, GSDMD inhibition in a cell can activate the interferon response $^{182}$. In a mouse study that modelled systemic lupus erythematosus (SLE) using injected imiquimod, the severity of SLE was unexpectedly exacerbated in $G s d m d^{-/-}$mice, leading to more severe kidney and pulmonary pathology, more autoantibodies and reduced mortality ${ }^{183}$. Type I interferons play an important driving role in many SLE models and in patients with SLE. GSDMD may have suppressed interferon induction in these mice, although a change in interferon signalling in this SLE model in Gsdmd ${ }^{-/-}$mice was not examined.

Mouse models may not always accurately mirror human disease pathogenesis, and mouse models of inflammatory diseases can be affected by small differences in experimental protocol or differences in the microbiome of mouse colonies or other poorly defined environmental or genetic differences. For example, two recent studies of dextran sodium sulfate-induced colitis found opposite results: in one study Gsdmd deficiency exacerbated colitis and enhanced cGASSTING-mediated interferon induction ${ }^{184}$, while in another study lack of $G s d m d$ reduced disease severity ${ }^{185}$.

The role of GSDMD in various inflammatory diseases and potential interactions of GSDMD activation with other innate immune responses will need to be examined carefully to select disease indications and anticipate possible toxicity of GSDM-modulating compounds as they become available.

\section{Concluding remarks and future perspectives}

Since the initial discovery of GSDMD as the substrate of inflammatory caspases, much progress has been made in understanding the activation mechanisms of the GSDM family. Two ways to activate GSDMs have so far been identified: first, protease-mediated cleavage in the linker region to liberate the NT active domain, and second, CT domain mutations including many germline-encoded disease mutations that disrupt the interaction of the NT and CT domains and diminish the ability of the CT domain to inhibit NT domain pore formation (FICS 2-5). Although the physiological stimuli that activate GSDMs are still being uncovered, a theme of GSDMs as sensors of cytosolic protease activation has emerged. Currently these proteases include caspases, granule-associated proteases, such as neutrophil elastase and cathepsin G, and granzymes delivered to target cells by killer T cells. The flexibility and accessibility of the GSDM linker region increases its vulnerability to protease attack. Direct protease activation of a GSDM could induce inflammatory death in diverse types of cells beyond immune and barrier cells. It will not be surprising if GSDMs are found to be cleaved and activated by additional cellular proteases as well as by pathogen-encoded proteases.

As inflammation causes or contributes to many poorly controlled and sometimes lethal diseases and strongly augments antigen-specific immunity, there is a need to further elucidate the regulation of GSDMs at the root of inflammation. For example, inflammasome activation also exacerbates many mouse models of disease, including atherosclerosis, type II diabetes, gout, nonalcoholic steatohepatitis and several neurodegenerative diseases, including Alzheimer disease. GSDMs not only cause cell death, but are also required for unconventional protein secretion of the IL-1 family cytokines and other intracellular danger molecules such as HMGB1 that lack a secretion signal. What regulates whether GSDM activation causes lytic cell death or hyperactivation without cell death remains unclear. A related question is whether pyroptosis or hyperactivation generates a stronger innate immune signal. Factors released by GSDM activation must be quite different in pyroptotic versus hyperactive cells. There is no doubt that future studies will link the GSDM family to more physiological and pathological processes and uncover how these processes are controlled.

As the key perpetrators of inflammation, GSDMs are strategically situated for therapeutical manipulation of inflammation. Developing GSDM agonists or antagonists could transform the treatment of inflammatory disease and potentially lead to better vaccine adjuvants or immune therapy. So far, identifying specific compounds that inhibit GSDMs has been challenging. The currently available GSDMD inhibitors, NSA, DSF and DMF, all covalently modify reactive cysteines and are thus not completely specific. Further mechanistic understanding of GSDM pore formation and the structural intermediates that form in the transition from the inactive to the active state will undoubtedly inform drug identification and optimization. Similarly, understanding the molecular pathways that regulate pore formation could provide additional therapeutic targets. Given the crucial function of GSDMs in inflammation, the search for GSDM-modulating drugs is likely to be very active in the coming years.

Published online 10 March 2021
1. Tamura, M. et al. Members of a novel gene family, Gsdm, are expressed exclusively in the epithelium of the skin and gastrointestinal tract in a highly tissue-specific manner. Genomics 89, 618-629 (2007).

2. Shi, J., Gao, W. \& Shao, F. Pyroptosis: gasdermin mediated programmed necrotic cell death. Trends Biochem. Sci. 42, 245-254 (2017).

3. Liu, X. \& Lieberman, J. Knocking 'em dead: pore-forming proteins in immune defense. Annu. Rev. Immunol. 38, 455-485 (2020).

This is a comprehensive review of pore-forming proteins involved in host immunity.
4. Liu, X. \& Lieberman, J. A mechanistic understanding of pyroptosis: the fiery death triggered by invasive infection. Adv Immunol 135, 81-117 (2017).

5. Saeki, N., Kuwahara, Y., Sasaki, H., Satoh, H. \& Shiroishi, T. Gasdermin (Gsdm) localizing to mouse chromosome 11 is predominantly expressed in upper gastrointestinal tract but significantly suppressed in human gastric cancer cells. Mamm. Genome 11 718-724 (2000)

6. Van Laer, L. et al. Nonsyndromic hearing impairment is associated with a mutation in DFNA5. Nat. Genet. 20, 194-197 (1998)
7. Lammert, C. R. et al. AIM2 inflammasome surveillance of DNA damage shapes neurodevelopment. Nature 580, 647-652 (2020)

8. Liu, X. et al. Inflammasome-activated gasdermin D causes pyroptosis by forming membrane pores. Nature 535, 153-158 (2016).

9. Ding, J. et al. Pore-forming activity and structural autoinhibition of the gasdermin family. Nature $\mathbf{5 3 5}$ $111-116$ (2016)

10. Sborgi, L. et al. GSDMD membrane pore formation constitutes the mechanism of pyroptotic cell death EMBO J. 35, 1766-1778 (2016). 
11. Aglietti, R. A. et al. GsdmD p30 elicited by caspase-11 during pyroptosis forms pores in membranes. Proc. Natl Acad. Sci. USA 113, 7858-7863 (2016). Liu et al. (2016), Ding et al. (2016), Sborgi et al. (2016) and Aglietti et al. (2016) demonstrate that the NT domain of GSDMD induces pyroptosis by oligomerization in membranes to form pores.

12. Liu, Z. et al. Crystal structures of the full-length murine and human gasdermin D reveal mechanisms of autoinhibition, lipid binding, and oligomerization. Immunity 51, 43-49 e44 (2019).

13. Shi, J. et al. Cleavage of GSDMD by inflammatory caspases determines pyroptotic cell death. Nature 526, 660-665 (2015).

14. Kayagaki, N. et al. Caspase-11 cleaves gasdermin D for non-canonical inflammasome signalling. Nature 526, 666-671 (2015).

Shi et al. (2015) and Kayagaki et al. (2015) identify GSDMD as a key executioner of inflammasome-triggered pyroptosis.

15. He, W. T. et al. Gasdermin D is an executor of pyroptosis and required for interleukin- 1 beta secretion. Cell Res. 25, 1285-1298 (2015).

16. Rogers, C. et al. Cleavage of DFNA5 by caspase- 3 during apoptosis mediates progression to secondary necrotic/pyroptotic cell death. Nat. Commun. 8 14128 (2017).

17. Wang, Y. et al. Chemotherapy drugs induce pyroptosis through caspase-3 cleavage of a gasdermin. Nature 547, 99-103 (2017)

Rogers et al. (2017) and Wang et al. (2017) show that GSDME is activated during classical apoptosis by caspase 3-mediated cleavage after Asp270, which converts cell apoptosis into pyroptosis.

18. Zhang, Z. et al. Gasdermin E suppresses tumour growth by activating anti-tumour immunity. Nature 579, 415-420 (2020).

19. Zhou, Z. et al. Granzyme A from cytotoxic lymphocytes cleaves GSDMB to trigger pyroptosis in target cells. Science 368, eaaz7548 (2020).

20. Hou, J. et al. PD-L1-mediated gasdermin C expression switches apoptosis to pyroptosis in cancer cells and facilitates tumour necrosis. Nat. Cell Biol. 22, 1264-1275 (2020)

Zhang et al. (2020) and Zhou et al. (2020) show that cell death-inducing granzymes cleave gasdermins to trigger pyroptosis of cancer cells, which potentiates antitumour immunity.

21. Evavold, C. L. et al. The pore-forming protein gasdermin D regulates interleukin-1 secretion from living macrophages. Immunity 48, 35-44 e36 (2018).

22. Chen, X. et al. Pyroptosis is driven by non-selective gasdermin-D pore and its morphology is different from MLKL channel-mediated necroptosis. Cell Res. 26, 1007-1020 (2016)

23. Ruhl, S. et al. ESCRT-dependent membrane repair negatively regulates pyroptosis downstream of GSDMD activation. Science 362, 956-960 (2018). This paper shows that plasma membrane repair can remove GSDMD pores from the cell membrane to suppress pyroptosis after inflammasome activation.

24. Zanoni, I., Tan, Y., Di Gioia, M., Springstead, J. R. \& Kagan, J. C. By capturing inflammatory lipids released from dying cells, the receptor CD14 induces inflammasome-dependent phagocyte hyperactivation. Immunity 47, 697-709.e693 (2017).

25. Li, S. et al. Gasdermin D in peripheral myeloid cells drives neuroinflammation in experimental autoimmune encephalomyelitis. J. Exp. Med. 216, 2562-2581 (2019).

26. Xiao, J. et al. Gasdermin D mediates the pathogenesis of neonatal-onset multisystem inflammatory disease in mice. PLoS Biol. 16, e3000047 (2018).

27. Runkel, F. et al. The dominant alopecia phenotypes Bareskin, Rex-denuded, and Reduced Coat 2 are caused by mutations in gasdermin 3. Genomics 84 824-835 (2004).

28. Lunny, D. P. et al. Mutations in gasdermin 3 cause aberrant differentiation of the hair follicle and sebaceous gland. J. Invest. Dermatol. 124, 615-621 (2005).

29. Tanaka, S. et al. A new Gsdma3 mutation affecting anagen phase of first hair cycle. Biochem. Biophys. Res. Commun. 359, 902-907 (2007).

30. Li, J. et al. Gsdma3 is required for hair follicle differentiation in mice. Biochem. Biophys. Res. Commun. 403, 18-23 (2010).

31. Kumar, S. et al. Gsdma3(I359N) is a nove ENU-induced mutant mouse line for studying the function of gasdermin A3 in the hair follicle and epidermis. J. Dermatol. Sci. 67, 190-192 (2012).

32. Das, S. et al. GSDMB induces an asthma phenotype characterized by increased airway responsiveness and remodeling without lung inflammation. Proc. Natl Acad. Sci. USA 113, 13132-13137 (2016).

33. Yu, C. et al. A 3-nucleotide deletion in the polypyrimidine tract of intron 7 of the DFNA5 gene causes nonsyndromic hearing impairment in a Chinese family. Genomics 82, 575-579 (2003).

34. Bischoff, A. M. et al. A novel mutation identified in the DFNA5 gene in a Dutch family: a clinical and genetic evaluation. Audiol. Neurootol. 9, 34-46 (2004).

35. Cheng, J. et al. A novel DFNA5 mutation, IVS8+4 A>C in the splice donor site of intron 8 causes late-onset non-syndromic hearing loss in a Chinese family. Clin. Genet. 72, 471-477 (2007).

36. Park, H. J. et al. Evidence for a founder mutation causing DFNA5 hearing loss in East Asians. J. Hum Genet. 55, 59-62 (2010)

37. Nishio, A. et al. A DFNA5 mutation identified in Japanese families with autosomal dominant hereditary hearing loss. Ann. Hum. Genet. 78, 83-91 (2014).

38. Li-Yang, M. N. et al. IVS8+1 DelG, a novel splice site mutation causing DFNA5 deafness in a chinese family. Chin. Med. J. 128, 2510-2515 (2015).

39. Delmaghani, S. et al. Mutations in the gene encoding pejvakin, a newly identified protein of the afferent auditory pathway, cause DFNB59 auditory neuropathy. Nat. Genet. 38, 770-778 (2006)

40. Zhu, Q. et al. Promotes AIM2 inflammasome activation and is required for host protection against Francisella novicida. J. Immunol. 201, 3662-3668 (2018).

41. Thurston, T. L. et al. Growth inhibition of cytosolic Salmonella by caspase- 1 and caspase- 11 precedes host cell death. Nat. Commun. 7, 13292 (2016).

42. Cerqueira, D. M. et al. Guanylate-binding protein 5 licenses caspase- 11 for gasdermin-D mediated host resistance to Brucella abortus infection. PLoS Pathog 14, e1007519 (2018)

43. Sollberger, G. et al. Gasdermin D plays a vital role in the generation of neutrophil extracellular traps. Sci. Immunol. 3, eaar6689 (2018).

44. Kambara, H. et al. Gasdermin D exerts anti-inflammatory effects by promoting neutrophil death. Cell Rep. 22, 2924-2936 (2018).

Sollberger et al. (2018) and Kambara et al. (2018) highlight the essential role of GSDMD in the formation of NETs.

45. Rathkey, J. K. et al. Chemical disruption of the pyroptotic pore-forming protein gasdermin $\mathrm{D}$ inhibits inflammatory cell death and sepsis. Sci. Immunol. 3, eaat2738 (2018)

46. Goncalves, A. V. et al. Gasdermin-D and caspase-7 are the key caspase- $1 / 8$ substrates downstream of the NAIP5/NLRC4 inflammasome required for restriction of Legionella pneumophila. PLoS Pathog. 15 e1007886 (2019).

47. Zhang, Z., Zhang, Y. \& Lieberman, J. Lighting a fire: can we harness pyroptosis to ignite anti-tumor immunity? Cancer Immunol. Res. 9, 2-7 (2021).

48. Hu, J. J. et al. FDA-approved disulfiram inhibits pyroptosis by blocking gasdermin $\mathrm{D}$ pore formation Nat. Immunol. 21, 736-745 (2020).

This work identifies DSF - a drug used to treat alcoholism - as an effective inhibitor of GSDMD pore formation by modifying a reactive Cys in GSDMD

49. Humphries, F. et al. Succination inactivates gasdermin D and blocks pyroptosis. Science 369, 1633-1637 (2020).

50. Jiang, S., Zhou, Z., Sun, Y., Zhang, T. \& Sun, L. Coral gasdermin triggers pyroptosis. Sci. Immunol. 5, eabd2591 (2020).

51. Zhang, Z. \& Lieberman, J. Lighting a fire on the reef Sci Immunol. 5, eabf0905 (2020).

52. Saeki, N. et al. GASDERMIN, suppressed frequently in gastric cancer, is a target of LMO1 in TGF-betadependent apoptotic signalling. Oncogene 26, 6488-6498 (2007).

53. Kayagaki, N. et al. IRF2 transcriptionally induces GSDMD expression for pyroptosis. Sci. Signal. 12 eaax4917 (2019).

54. Webb, M. S., Miller, A. L. \& Thompson, E. B. In CEM cells the autosomal deafness gene dfna5 is regulated by glucocorticoids and forskolin. J. Steroid Biochem. Mol. Biol. 107, 15-21 (2007).

55. Moussette, S. et al. Role of DNA methylation in expression control of the IKZF3-GSDMA region in human epithelial cells. PLOS ONE 12, e0172707 (2017).
56. Akino, K. et al. Identification of DFNA5 as a target of epigenetic inactivation in gastric cancer. Cancer $\mathrm{Sci}$. 98, 88-95 (2007)

57. Kim, M. S. et al. Methylation of the DFNA5 increases risk of lymph node metastasis in human breast cancer. Biochem. Biophys. Res. Commun. 370, 38-43 (2008).

58. Croes, L. et al. DFNA5 promoter methylation a marker for breast tumorigenesis. Oncotarget 8 31948-31958 (2017).

59. Croes, L. et al. Large-scale analysis of DFNA5 methylation reveals its potential as biomarker for breast cancer. Clin. Epigenetics 10, 51 (2018).

60. Kim, M. S. et al. Aberrant promoter methylation and tumor suppressive activity of the DFNA5 gene in colorectal carcinoma. Oncogene 27, 3624-3634 (2008).

61. Sato, H. et al. A new mutation Rim3 resembling $\mathrm{Re}(d e n)$ is mapped close to retinoic acid receptor alpha (Rara) gene on mouse chromosome 11 . Mamm. Genome 9, 20-25 (1998).

62. Katoh, M. \& Katoh, M. Evolutionary recombination hotspot around GSDML-GSDM locus is closely linked to the oncogenomic recombination hotspot around the PPP1R1B-ERBB2-GRB7 amplicon. Int. J. Oncol. 24, 757-763 (2004).

63. Lei, M. et al. Gsdma3 is a new factor needed for TNF-alpha-mediated apoptosis signal pathway in mouse skin keratinocytes. Histochem. Cell Biol. 138, 385-396 (2012)

64. Tanaka, S., Mizushina, Y., Kato, Y., Tamura, M. \& Shiroishi, T. Functional conservation of Gsdma cluster genes specifically duplicated in the mouse genome. G3 3, 1843-1850 (2013)

65. Shi, P. et al. Loss of conserved Gsdma3 self-regulation causes autophagy and cell death. Biochem. J. 468, 325-336 (2015)

66. Soderman, J., Berglind, L. \& Almer, S. Gene expression-genotype analysis implicates GSDMA, GSDMB, and LRRC3C as contributors to inflammatory bowel disease susceptibility. BioMed. Res. Int. 2015 834805 (2015).

67. Moreno-Moral, A. et al. Changes in macrophage transcriptome associate with systemic sclerosis and mediate GSDMA contribution to disease risk. Ann. Rheum. Dis. 77, 596-601 (2018)

68. Terao, C. et al. Transethnic meta-analysis identifies GSDMA and PRDM 1 as susceptibility genes to systemic sclerosis. Ann. Rheum. Dis. 76, 1150-1158 (2017).

69. Yu, J. et al. Polymorphisms in GSDMA and GSDMB are associated with asthma susceptibility, atopy and BHR. Pediatr. Pulm. 46, 701-708 (2011).

70. Saeki, N. et al. Distinctive expression and function of four GSDM family genes (GSDMA-D) in normal and malignant upper gastrointestinal epithelium. Genes Chromosomes Cancer 48, 261-271 (2009)

71. Carl-McGrath, S., Schneider-Stock, R., Ebert, M. \& Rocken, C. Differential expression and localisation of gasdermin-like (GSDML), a novel member of the cancer-associated GSDMDC protein family, in neoplastic and non-neoplastic gastric, hepatic, and colon tissues. Pathology 40, 13-24 (2008).

72. Hu, Y., Jin, S., Cheng, L., Liu, G. \& Jiang, Q Autoimmune disease variants regulate GSDMB gene expression in human immune cells and whole blood. Proc. Natl Acad. Sci. USA 114, E7860-E7862 (2017).

73. Chen, Q. et al. GSDMB promotes non-canonical pyroptosis by enhancing caspase- 4 activity. J. Mol. Cell Biol. 11, 496-508 (2019).

74. Verlaan, D. J. et al. Allele-specific chromatin remodeling in the ZPBP2/CSDMB/ORMDL3 locus associated with the risk of asthma and autoimmune disease. Am. J. Hum. Genet. 85, 377-393 (2009).

75. $\mathrm{Wu}, \mathrm{H}$. et al. Genetic variation in ORM1-like 3 (ORMDL3) and gasdermin-like (GSDML) and childhood asthma. Allergy 64, 629-635 (2009).

76. Kang, M. J. et al. GSDMB/ORMDL3 variants contribute to asthma susceptibility and eosinophilmediated bronchial hyperresponsiveness. Hum. Immunol. 73, 954-959 (2012).

77. Moffatt, M. F. et al. A large-scale, consortium-based genomewide association study of asthma. N. Engl. J. Med. 363, 1211-1221 (2010).

78. Hergueta-Redondo, M. et al. Gasdermin-B promotes invasion and metastasis in breast cancer cells. PLOS ONE 9, e90099 (2014).

79. Sun, Q. et al. Expression of GSDML associates with tumor progression in uterine cervix cancer. Transl. Oncol. 1, 73-83 (2008). 
80. Hergueta-Redondo, M. et al. Gasdermin B expression predicts poor clinical outcome in HER2-positive breast cancer. Oncotarget 7, 56295-56308 (2016).

81. Katoh, M. \& Katoh, M. Identification and characterization of human DFNA5L, mouse Dfna5I, and rat Dfna5I genes in silico. Int. J. Oncol. 25, 765-770 (2004).

82. Watabe, K. et al. Structure, expression and chromosome mapping of MLZE, a novel gene which is preferentially expressed in metastatic melanoma cells. Jpn. J. Cancer Res. 92, 140-151 (2001).

83. Kusumaningrum, N., Lee, D. H., Yoon, H. S., Park, C. H. $£$ Chung, J. H. Ultraviolet light-induced gasdermin C expression is mediated via TRPV1/calcium/calcineurin/ NFATC1 signaling. Int. J. Mol. Med. 42, 2859-2866 (2018).

84. Kusumaningrum, N. et al. Gasdermin $\mathrm{C}$ is induced by ultraviolet light and contributes to MMP-1 expression via activation of ERK and JNK pathways. J. Dermatol. Sci. 90, 180-189 (2018).

85. Miguchi, M. et al. Gasdermin C is upregulated by inactivation of transforming growth factor $\beta$ recepto type II in the presence of mutated Apc, promoting colorectal cancer proliferation. PLOS ONE 11 e0166422 (2016).

86. Agard, N. J., Maltby, D. \& Wells, J. A. Inflammatory stimuli regulate caspase substrate profiles. Mol. Cell Proteom. 9, 880-893 (2010).

87. Fujii, T. et al. Gasdermin D (Gsdmd) is dispensable for mouse intestinal epithelium development. Genesis 46 418-423 (2008)

88. Rathkey, J. K., Xiao, T. S. \& Abbott, D. W. Human polymorphisms in CSDMD alter the inflammatory response. J. Biol. Chem. 295, 3228-3238 (2020).

89. Wang, J., Deobald, K. \& Re, F. Gasdermin D protects from melioidosis through pyroptosis and direct killing of bacteria. J. Immunol. 202, 3468-3473 (2019).

90. Dubois, $\mathrm{H}$. et al. NIrp3 inflammasome activation and gasdermin D-driven pyroptosis are immunopathogenic upon gastrointestinal norovirus infection. PLoS Pathog. 15, e1007709 (2019).

91. de Beeck, K. O., Van Laer, L. \& Van Camp, G. DFNA5, a gene involved in hearing loss and cancer: a review. Ann. Otol. Rhinol. Laryngol. 121, 197-207 (2012).

92. Thompson, D. A. \& Weigel, R. J. Characterization of a gene that is inversely correlated with estrogen receptor expression (ICERE-1) in breast carcinomas. Eur. J. Biochem. 252, 169-177 (1998).

93. Masuda, Y. et al. The potential role of DFNA5, a hearing impairment gene, in p53-mediated cellular response to DNA damage. J. Hum. Genet. 51, 652-664 (2006)

94. Yokomizo, K. et al. Methylation of the DFNA5 gene is frequently detected in colorectal cancer. Anticancer. Res. 32, 1319-1322 (2012).

95. Stoll, G. et al. Pro-necrotic molecules impact local immunosurveillance in human breast cancer. Oncoimmunology 6, e1299302 (2017).

96. Delmaghani, S. et al. Hypervulnerability to sound exposure through impaired adaptive proliferation of peroxisomes. Cell 163, 894-906 (2015).

97. Schwander, M et al. A forward genetics screen in mice identifies recessive deafness traits and reveals that pejvakin is essential for outer hair cell function. J. Neurosci. 27, 2163-2175 (2007).

98. Van Laer, L. et al. Mice lacking Dfna5 show a diverging number of cochlear fourth row outer hair cells. Neurobiol Dis 19,386-399 (2005).

99. Harris, S. L. et al. Conditional deletion of pejvakin in adult outer hair cells causes progressive hearing loss in mice. Neuroscience 344, 380-393 (2017).

100. Defourny, J. et al. Pejvakin-mediated pexophagy protects auditory hair cells against noise-induced damage. Proc. Natl Acad. Sci. USA 116, 8010-8017 (2019).

101. Bauernfeind, F. G. et al. Cutting edge: NF-kB activating pattern recognition and cytokine receptors license NLRP3 inflammasome activation by regulating NLRP3 expression. J. Immunol. 182, 787-791 (2009).

102. Black, R. A. et al. Generation of biologically active interleukin- 1 beta by proteolytic cleavage of the inactive precursor. J. Biol. Chem. 263, 9437-9442 (1988).

103. Thornberry, N. A. et al. A novel heterodimeric cysteine protease is required for interleukin-1 beta processing in monocytes. Nature 356, 768-774 (1992).

104. Kayagaki, N. et al. Non-canonical inflammasome activation targets caspase-11. Nature 479, 117-121 (2011)

This study demonstrates the pro-inflammatory role of caspase 11 in response to Gram-negative bacterial infection.
105. Orning, P. et al. Pathogen blockade of TAK1 triggers caspase-8-dependent cleavage of gasdermin $\mathrm{D}$ and cell death. Science 362, 1064-1069 (2018).

106. Sarhan, J. et al. Caspase-8 induces cleavage of gasdermin $D$ to elicit pyroptosis during Yersinia infection. Proc. Natl Acad. Sci. USA 115,

E10888-E10897 (2018)

Orning et al. (2018) and Sarhan et al. (2018) describe an alternative GSDMD cleavage and activation pathway, which is mediated by caspase 8 when TAK 1 is inhibited by the Yersinia effector protein YopJ.

107. Demarco, B. et al. Caspase-8-dependent gasdermin D cleavage promotes antimicrobial defense but confers susceptibility to TNF-induced lethality. Sci. Adv. 6 eabc3465 (2020).

108. Schwarzer, R., Jiao, H., Wachsmuth, L., Tresch, A $\&$ Pasparakis, M. FADD and Caspase- 8 regulate gut homeostasis and inflammation by controlling MLKL- and GSDMD-mediated death of intestinal epithelial cells. Immunity 52, 978-993 (2020).

109. Muendlein, H. I. et al. cFLIPL protects macrophages from LPS-induced pyroptosis via inhibition of complex II formation. Science 367, 1379-1384 (2020)

110. Taabazuing, C. Y., Okondo, M. C. \& Bachovchin, D. A. Pyroptosis and apoptosis pathways engage in bidirectional crosstalk in monocytes and macrophages. Cell Chem. Biol. 24, 507-514 e504 (2017).

111. Chowdhury, D. \& Lieberman, J. Death by a thousand cuts: granzyme pathways of programmed cell death Annu. Rev. Immunol. 26, 389-420 (2008)

112. Heusel, J. W., Wesselschmidt, R. L., Shresta, S. Russell, J. H. \& Ley, T. J. Cytotoxic lymphocytes require granzyme $B$ for the rapid induction of DNA fragmentation and apoptosis in allogeneic target cells. Cell 76, 977-987 (1994).

113. Darmon, A. J., Nicholson, D. W. \& Bleackley, R. C. Activation of the apoptotic protease CPP32 by cytotoxic T-cell-derived granzyme B. Nature 377, 446-448 (1995)

114. Yang, J. et al. Mechanism of gasdermin D recognition by inflammatory caspases and their inhibition by a gasdermin D-derived peptide inhibitor. Proc. Natl Acad. Sci. USA 115, 6792-6797 (2018).

115. Wang, K. et al. Structural mechanism for GSDMD targeting by autoprocessed caspases in pyroptosis. Cell 180, 941-955 e920 (2020).

116. Liu, Z. et al. Caspase-1 engages full-length gasdermin $D$ through two distinct interfaces that mediate caspas recruitment and substrate cleavage. Immunity $\mathbf{5 3}$ 106-114 (2020).

117. Ruan, J., Xia, S., Liu, X., Lieberman, J. \& Wu, H Cryo-EM structure of the gasdermin A3 membrane pore. Nature 557, 62-67 (2018) This study describes GSDMA3 membrane pore structure resolved by cryo-EM

118. Mulvihill, E. et al. Mechanism of membrane pore formation by human gasdermin-D. EMBO J. 37 , e98321 (2018).

119. Rogers, C. et al. Gasdermin pores permeabilize mitochondria to augment caspase- 3 activation during apoptosis and inflammasome activation. Nat. Commun. 10, 1689 (2019).

120. Zanoni, I. et al. An endogenous caspase-11 ligand elicits interleukin-1 release from living dendritic cells. Science 352, 1232-1236 (2016).

Zanoni et al. (2016), Zanoni et al. (2017) and Evavold et al. (2018) show that inflammasome activation does not always cause pyroptosis, but surviving cells still secrete IL-1 family cytokines.

121. Wolf, A. J. et al. Hexokinase is an innate immune receptor for the detection of bacterial peptidoglycan. Cell 166, 624-636 (2016).

122. Heilig, R. et al. The gasdermin-D pore acts as a conduit for IL-1 $\beta$ secretion in mice. Eur. J. Immunol. 48, 584-592 (2018)

123. Gaidt, M. M. et al. Human monocytes engage an alternative inflammasome pathway. Immunity 44 833-846 (2016)

124. Carty, M. et al. Cell survival and cytokine release after inflammasome activation is regulated by the Toll-IL-1R protein SARM. Immunity 50, 1412-1424.e1416 (2019).

125. Chen, K. W. et al. The neutrophil NLRC4 inflammasome selectively promotes IL- 1 beta maturation without pyroptosis during acute Salmonella challenge. Cell Rep. 8, 570-582 (2014).

126. Karmakar, M., Katsnelson, M. A., Dubyak, G. R. \& Pearlman, E. Neutrophil P2X7 receptors mediate NLRP3 inflammasome-dependent IL- $1 \beta$ secretion in response to ATP. Nat. Commun. 7, 10555 (2016).
127. Karmakar, M. et al. N-GSDMD trafficking to neutrophil organelles facilitates IL-1 beta release independently of plasma membrane pores and pyroptosis. Nat. Commun. 11, 2212 (2020).

128. McNeil, P. L. \& Kirchhausen, T. An emergency response team for membrane repair. Nat Rev. Mol. Cell Biol. 6, 499-505 (2005).

129. Monteleone, M. et al. Interleukin- $1 \beta$ maturation triggers its relocation to the plasma membrane for gasdermin-D-dependent and -independent secretion. Cell Rep. 24, 1425-1433 (2018).

130. Lin, P. H. Lin, H. Y. Kuo C. C. \& Yang L. T. N-terminal functional domain of gasdermin $\mathrm{A} 3$ regulates mitochondrial homeostasis via mitochondria targeting. J. Biomed. Sci. 22, 44 (2015).

131. Chen, K. W. et al. Noncanonical inflammasome signaling elicits gasdermin D-dependent neutrophil extracellular traps. Sci. Immunol. 3, eaar6676 (2018)

132. Schlame, M. Cardiolipin synthesis for the assembly of bacterial and mitochondrial membranes. J. Lipid Res. 49, 1607-1620 (2008)

133. Chao, K. L., Kulakova, L. \& Herzberg, O. Gene polymorphism linked to increased asthma and IBD risk alters gasdermin-B structure, a sulfatide and phosphoinositide binding protein. Proc. Natl Acad. Sci. USA 114, E1128-E1137 (2017).

134. Papayannopoulos, V. Neutrophil extracellular traps in immunity and disease. Nat. Rev. Immunol. 18 134-147 (2018)

135. Burgener, S. S. et al. Cathepsin G inhibition by Serpinb 1 and Serpinb6 prevents programmed necrosis in neutrophils and monocytes and reduces GSDMD-driven inflammation. Cell Rep. 27. 3646-3656 e3645 (2019).

136. Beckwith, K. S. et al. Plasma membrane damage causes NLRP3 activation and pyroptosis during Mycobacterium tuberculosis infection. Nat. Commun. 11, 2270 (2020)

137. Fisch, D. et al. Human GBP1 is a microbe-specific gatekeeper of macrophage apoptosis and pyroptosis. EMBO J. 38, e100926 (2019).

138. Pandori, W. J. et al. Toxoplasma gondii activates a Syk-CARD9-NF-kappaB signaling axis and gasdermin D-independent release of IL- 1 beta during infection of primary human monocytes. PLoS Pathog. 15, e1007923 (2019).

139. Batista, S. J. et al. Gasdermin-D-dependent IL-1 alpha release from microglia promotes protective immunity during chronic Toxoplasma gondii infection. Nat. Commun. 11, 3687 (2020)

140. Quach, J., Moreau, F., Sandall, C. \& Chadee, K. Entamoeba histolytica-induced IL-1 beta secretion is dependent on caspase- 4 and gasdermin $D$. Mucosal Immunol. 12, 323-339 (2019).

141. Zhu, S. et al. Nlrp9b inflammasome restricts rotavirus infection in intestinal epithelial cells. Nature $\mathbf{5 4 6}$ 667-670 (2017).

142. Liu, T. et al. NOD-like receptor family, pyrin domain containing 3 (NLRP3) contributes to inflammation, pyroptosis, and mucin production in human airway epithelium on rhinovirus infection. J. Allergy Clin. Immunol. 144, 777-787 e779 (2019).

143. Broz, P. \& Dixit, V. M. Inflammasomes: mechanism of assembly, regulation and signalling. Nat. Rev. Immunol. 16, 407-420 (2016). This is a comprehensive review of inflammasome signalling.

144. Kanneganti, A et al. GSDMD is critical for autoinflammatory pathology in a mouse model of familial mediterranean fever. J. Exp. Med. 215 1519-1529 (2018).

145. O'Neill, L. A. The interleukin-1 receptor/Toll-like receptor superfamily: signal transduction during inflammation and host defense. SCi. STKE 2000, re1 (2000).

146. Yang, X. et al. Bacterial endotoxin activates the coagulation cascade through gasdermin D-dependent phosphatidylserine exposure. Immunity 51, 983-996 e986 (2019)

147. Wu, C. et al. Inflammasome activation triggers blood clotting and host death through pyroptosis. Immunity 50, 1401-1411 e1404 (2019).

148. McDonald, B. et al. Platelets and neutrophil extracellular traps collaborate to promote intravascular coagulation during sepsis in mice. Blood 129, 1357-1367 (2017).

149. Kayagaki, N. et al. Noncanonical inflammasome activation by intracellular LPS independent of TLR4 Science 341, 1246-1249 (2013). This work demonstrates that cytosolic LPS triggers a caspase 11-dependent noncanonical inflammasome pathway. 
150. Chen, H. et al. RIPK3 collaborates with GSDMD to drive tissue injury in lethal polymicrobial sepsis. Cell Death Differ. 27, 2568-2585 (2020)

151. McKenzie, B. A. et al. Caspase-1 inhibition prevents glial inflammasome activation and pyroptosis in models of multiple sclerosis. Proc. Natl Acad. Sci. USA 115, E6065-E6074 (2018).

152. Xiao, J. et al. Radiation causes tissue damage by dysregulating inflammasome-gasdermin D signaling in both host and transplanted cells. PLOS Biol. 18 e3000807 (2020).

153. Lu, Y. et al. Caspase- 11 signaling enhances graft-versus-host disease. Nat. Commun. 10, 4044 (2019).

154. Xu, B. et al. Gasdermin D plays a key role as a pyroptosis executor of non-alcoholic steatohepatitis in humans and mice. J. Hepatol. 68, 773-782 (2018).

155. Khanova, E. et al. Pyroptosis by caspase 11/4-gasdermin-D pathway in alcoholic hepatitis in mice and patients. Hepatology 67, 1737-1753 (2018)

156. Yang, C et al. Gasdermin D protects against noninfectious liver injury by regulating apoptosis and necroptosis. Cell Death Dis. 10, 481 (2019).

157. Li, J. et al. Blocking GSDMD processing in innate immune cells but not in hepatocytes protects hepatic ischemia-reperfusion injury. Cell Death Dis. 11, 244 (2020).

158. Zhang, D. et al. Gasdermin D serves as a key executioner of pyroptosis in experimental cerebra ischemia and reperfusion model both in vivo and in vitro. J. Neurosci. Res. 97, 645-660 (2019).

159. Jia, C. et al. Endothelial cell pyroptosis plays an important role in Kawasaki disease via HMGB 1 RAGE/cathespin B signaling pathway and NLRP3 inflammasome activation. Cell Death Dis. 10, 778 (2019).

160. Wang, Y. et al. TLR4/NF-kappaB signaling induces GSDMD-related pyroptosis in tubular cells in diabetic kidney disease. Front. Endocrinol. 10, 603 (2019).

161. Yang, F. et al. Silencing long non-coding RNA Kcnq 1 ot 1 alleviates pyroptosis and fibrosis in diabetic cardiomyopathy. Cell Death Dis. 9 1000 (2018)

162. Zhang, Z. et al. Caspase-11-mediated tubular epithelial pyroptosis underlies contrast-induced acute kidney injury. Cell Death Dis. 9, 983 (2018)

163. Yuan, B. et al. Inhibition of AIM2 inflammasome activation alleviates GSDMD-induced pyroptosis in early brain injury after subarachnoid haemorrhage. Cell Death Dis. 11, 76 (2020).

164. Hu, X. et al. Role of pyroptosis in traumatic brain and spinal cord injuries. Int. J. Biol. Sci. 16, 2042-2050 (2020).

165. Wang, W. J. et al. Downregulation of gasdermin D promotes gastric cancer proliferation by regulating cell cycle-related proteins. J. Dig. Dis. 19, 74-83 (2018).

166. Gao, J. et al. Downregulation of GSDMD attenuates tumor proliferation via the intrinsic mitochondrial apoptotic pathway and inhibition of EGFR/Akt signaling and predicts a good prognosis in nonsmall cell lung cancer. Oncol. Rep. 40, 1971-1984 (2018).

167. Molina-Crespo, A. et al. Intracellular delivery of an antibody targeting gasdermin-B reduces HER2 breast cancer aggressiveness. Clin. Cancer Res. 25 4846-4858 (2019)

168. Wei, J. et al. Overexpression of GSDMC is a prognostic factor for predicting a poor outcome in lung adenocarcinoma. Mol. Med. Rep. 21, 360-370 (2020).
169. Grivennikov, S. I., Greten, F. R. \& Karin, M. Immunity, inflammation, and cancer. Cell 140, 883-899 (2010).

170. Lage, H., Helmbach, H., Grottke, C., Dietel, M. \& Schadendorf, D. DFNA5 (ICERE-1) contributes to acquired etoposide resistance in melanoma cells. FEBS Lett. 494, 54-59 (2001).

171. Lu, H. et al. Molecular targeted therapies elicit concurrent apoptotic and GSDME-dependent pyroptotic tumor cell death. Clin. Cancer Res. 24 6066-6077 (2018).

172. Liu, Y. et al. Gasdermin E-mediated target cell pyroptosis by CAR T cells triggers cytokine release syndrome. Sci. Immunol. 5, eaax7969 (2020).

173. Fan, J. X. et al. Epigenetics-based tumor cells pyroptosis for enhancing the immunological effect of chemotherapeutic nanocarriers. Nano Lett. 19 8049-8058 (2019).

174. Erkes, D. A. et al. Mutant BRAF and MEK inhibitors regulate the tumor immune microenvironment via pyroptosis. Cancer Discov. 10, 254-269 (2020).

175. Johnson, D. C. et al. DPP8/DPP9 inhibitor-induced pyroptosis for treatment of acute myeloid leukemia. Nat. Med. 24, 1151-1156 (2018).

176. Dinarello, C. A., Simon, A. \& van der Meer, J. W. Treating inflammation by blocking interleukin-1 in a broad spectrum of diseases. Nat. Rev. Drug Discov. $11,633-652(2012)$

177. Latz, E., Xiao, T. S. \& Stutz, A. Activation and regulation of the inflammasomes. Nat. Rev. Immunol. 13, 397-411 (2013)

178. Skrott, Z. et al. Alcohol-abuse drug disulfiram targets cancer via p97 segregase adaptor NPL4. Nature 552 194-199 (2017)

179. Martin-Sanchez, F. et al. Inflammasome-dependent IL-1 beta release depends upon membrane permeabilisation. Cell Death Differ. 23, 1219-1231 (2016).

180. Wang, Q. et al. A bioorthogonal system reveals antitumour immune function of pyroptosis. Nature 579, 421-426 (2020).

181. Rashidi, M. et al. The pyroptotic cell death effector gasdermin D is activated by gout-associated uric acid crystals but is dispensable for cell death and IL- 1 beta release. J. Immunol. 203, 736-748 (2019).

182. Banerjee, I. et al. Gasdermin D restrains type I interferon response to cytosolic DNA by disrupting ionic homeostasis. Immunity 49, 413-426 e415 (2018).

183. Wang, X. et al. Effects of gasdermin D in modulating murine lupus and its associated organ damage. Arthritis Rheumatol. 72, 2118-2129 (2020).

184. Ma, C. et al. Gasdermin D in macrophages restrains colitis by controlling cGAS-mediated inflammation. Sci. Adv. 6, eaaz6717 (2020).

185. Bulek, K. et al. Epithelial-derived gasdermin D mediates nonlytic IL-1 beta release during experimental colitis. J. Clin. Invest. 130, 4218-4234 (2020).

186. Fink, S. L. \& Cookson, B. T. Pyroptosis and host cell death responses during Salmonella infection. Cell Microbiol. 9, 2562-2570 (2007)

187. Zychlinsky, A., Prevost, M. C. \& Sansonetti, P. J. Shigella flexneri induces apoptosis in infected macrophages. Nature 358, 167-169 (1992).

188. Lindgren, S. W., Stojiljkovic, I. \& Heffron, F. Macrophage killing is an essential virulence mechanism of Salmonella typhimurium. Proc. Natl Acad. Sci. USA 93, 4197-4201 (1996)

189. Monack, D. M., Raupach, B. Hromockyj, A. E. \& Falkow, S. Salmonella typhimurium invasion induces apoptosis in infected macrophages. Proc. Natl Acad. Sci. USA 93, 9833-9838 (1996).
190. Chen, Y., Smith, M. R., Thirumalai, K. \& Zychlinsky, A A bacterial invasin induces macrophage apoptosis by binding directly to ICE. EMBO J. 15, 3853-3860 (1996).

191. Hilbi, H. et al. Shigella-induced apoptosis is dependent on caspase- 1 which binds to IpaB. J. Biol. Chem. 273 32895-32900 (1998).

192. Hersh, D. et al. The Salmonella invasin SipB induces macrophage apoptosis by binding to caspase- 1 . Proc. Natl Acad. Sci. USA 96, 2396-2401 (1999).

193. Zychlinsky, A., Fitting, C., Cavaillon, J. M. \& Sansonetti, P. J. Interleukin 1 is released by murine macrophages during apoptosis induced by Shigella flexneri. J. Clin. Invest. 94, 1328-1332 (1994).

194. Fernandez-Prada, C. M., Hoover, D. L., Tall, B. D. $\&$ Venkatesan, M. M. Human monocyte-derived macrophages infected with virulent Shigella flexner in vitro undergo a rapid cytolytic event similar to oncosis but not apoptosis. Infect. Immun. 65 1486-1496 (1997).

195. Brennan, M. A. \& Cookson, B. T. Salmonella induces macrophage death by caspase-1-dependent necrosis. Mol. Microbiol. 38, 31-40 (2000).

196. Cookson, B. T. \& Brennan, M. A. Pro-inflammatory programmed cell death. Trends Microbiol. 9, 113-114 (2001).

This paper proposes the term pyroptosis to describe inflammatory caspase-mediated cell death.

197. Taylor, R. C., Cullen, S. P. \& Martin, S. J. Apoptosis controlled demolition at the cellular level. Nat. Rev. Mol. Cell Biol. 9, 231-241 (2008).

198. Riedl, S. J. \& Shi, Y. Molecular mechanisms of caspase regulation during apoptosis. Nat. Rev. Mol. Cell Biol. 5, 897-907 (2004).

199. Thomas, M. P. \& Lieberman, J. Live or let die: posttranscriptional gene regulation in cell stress and cell death. Immunol. Rev. 253, 237-252 (2013).

200. Thomas, M. P. et al. Apoptosis triggers specific, rapid, and global mRNA decay with 3' uridylated intermediates degraded by DIS3L2. Cell Rep. 11 1079-1089 (2015)

201. Liu, X. et al. PNPT1 release from mitochondria during apoptosis triggers decay of poly(A) RNAs. Cell 174, 187-201 e 112 (2018)

202. Danial, N. N. \& Korsmeyer, S. J. Cell death: critical control points. Cell 116, 205-219 (2004).

203. Savill, J., Dransfield, I., Gregory, C. \& Haslett, C. A blast from the past: clearance of apoptotic cells regulates immune responses. Nat. Rev. Immunol. 2 965-975 (2002).

\section{Acknowledgements}

This work was supported by NIH R01Al139914 (H.W.), R01CA240955 (J.L.), Key Research Program of the Chinese Academy of Sciences (ZDBS-LY-SM008), National Key R\&D Program of China (2020YFA0509600), National Natural Science Foundation of China (31972897), Strategic Priority Research Program of Chinese Academy of Sciences (XDB29030300) and Shanghai Municipal Science and Technology Major Project (2019SHZDZX02) (X.L.).

Competing interests

H.W. and J.L. are co-founders of Ventus Therapeutics. The other authors declare no competing interests.

\section{Publisher's note}

Springer Nature remains neutral with regard to jurisdictional claims in published maps and institutional affiliations.

(c) Springer Nature Limited 2021 